\title{
Flood-Risk Analysis on Terrains under the Multiflow-Direction Model
}

\author{
AARON LOWE and PANKAJ K. AGARWAL, Duke University
}

\begin{abstract}
An important problem in terrain analysis is modeling how water flows across a terrain and creates floods by filling up depressions. In this article, we study a number of flood-risk related problems: Given a terrain $\Sigma$, represented as a triangulated $x y$-monotone surface with $n$ vertices, a rain distribution $\mathcal{R}$, and a volume of rain $\psi$, determine which portions of $\Sigma$ are flooded. We develop efficient algorithms for flood-risk analysis under the multiflow-directions (MFD) model, in which water at a point can flow along multiple downslope edges and which more accurately represent flooding events.

We present three main results: First, we present an $O(n \log n)$-time algorithm to answer a terrain-flood query: if it rains a volume $\psi$ according to a rain distribution $\mathcal{R}$, determine what regions of $\Sigma$ will be flooded. Second, we present a $O(n \log n+n m)$-time algorithm for preprocessing $\Sigma$ containing $m$ sinks into a data structure of size $O(\mathrm{~nm})$ for answering point-flood queries: Given a rain distribution $\mathcal{R}$, a volume of rain $\psi$ falling according to $\mathcal{R}$, and point $q \in \Sigma$, determine whether $q$ will be flooded. A point-flood query can be answered in $O\left(|\mathcal{R}| k+k^{2}\right)$ time, where $k$ is the number of maximal depressions in $\Sigma$ containing the query point $q$ and $|\mathcal{R}|$ is the number of vertices in $\mathcal{R}$ with positive rainfall. Finally, we present algorithms for answering a flood-time query: given a rain distribution $\mathcal{R}$ and a point $q \in \Sigma$, determine the volume of rain that must fall before $q$ is flooded. Assuming that the product of two $k \times k$ matrices can be computed in $O\left(k^{\omega}\right)$ time, we show that a flood-time query can be answered in $O\left(n k+k^{\omega}\right)$ time. We also give an $\alpha$-approximation algorithm, for $\alpha>1$, which runs in $O\left(n \log n \log _{\alpha} \rho\right)$-time, where $\rho$ is a variable on the terrain that depends on the ratio between depression volumes. We implemented our algorithms for computing terrain and point-flood queries as well as approximate flood-time queries. We tested the efficacy and efficiency of these algorithms on three real terrains of different types (urban, suburban, and mountainous.)
\end{abstract}

\section{CCS Concepts: • Information systems $\rightarrow$ Geographic information systems;}

Additional Key Words and Phrases: Terrains, flood-risk analysis, merge trees

\section{ACM Reference format:}

Aaron Lowe and Pankaj K. Agarwal. 2019. Flood-Risk Analysis on Terrains under the Multiflow-Direction Model. ACM Trans. Spatial Algorithms Syst. 5, 4, Article 26 (September 2019), 27 pages.

https://doi.org/10.1145/3340707

\section{INTRODUCTION}

An important problem in terrain analysis is modeling how water flows across a terrain and creates floods by filling up depressions. When rains fall, the rate at which a depression fills up depends

Work by Lowe and Agarwal is supported by NSF under grants CCF-15-13816, CCF-15-46392, and IIS-14-08846, by ARO grant W911NF-15-1-0408, and by Grant 2012/229 from the U.S.-Israel Binational Science Foundation.

Authors' address: A. Lowe and P. K. Agarwal, Department of Computer Science, LSRC Building, 308 Research Drive, Duke University, Box 90129, Durham, NC 27708-0129; emails: aaron@cs.duke.edu, pankaj@duke.edu.

Permission to make digital or hard copies of all or part of this work for personal or classroom use is granted without fee provided that copies are not made or distributed for profit or commercial advantage and that copies bear this notice and the full citation on the first page. Copyrights for components of this work owned by others than the author(s) must be honored. Abstracting with credit is permitted. To copy otherwise, or republish, to post on servers or to redistribute to lists, requires prior specific permission and/or a fee. Request permissions from permissions@acm.org.

(C) 2019 Copyright held by the owner/author(s). Publication rights licensed to ACM.

2374-0353/2019/09-ART26 \$15.00

https://doi.org/10.1145/3340707 


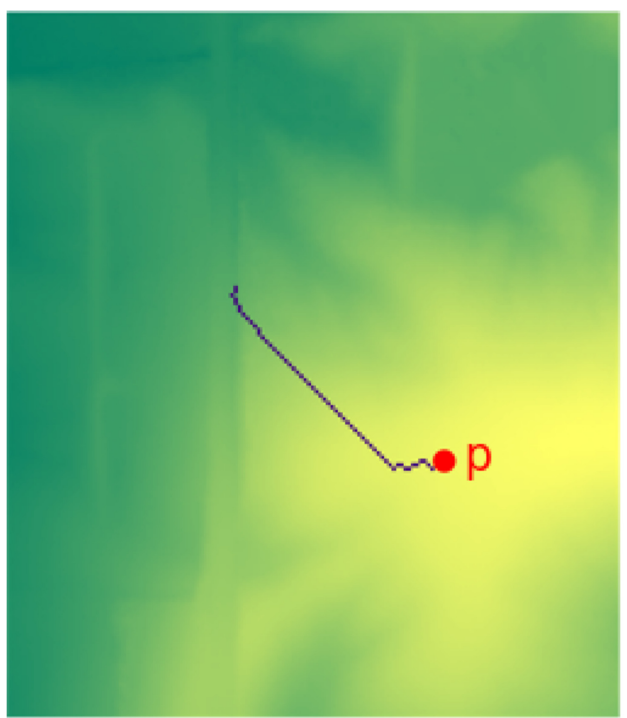

(a)

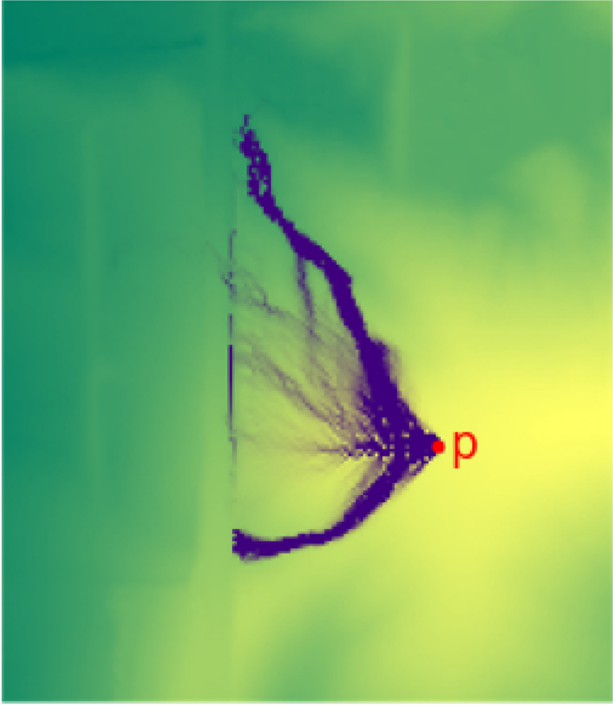

(b)

Fig. 1. Rain falls at point $p$ on the terrain, with color indicating relative height, with darker areas being lower. (a) Under SFD, water follows a single path, highlighted in blue, until it reaches a sink (b) Under MFD, it splits and flows to many sinks, with darker shades of blue denoting more water flowing over that point.

not only on its shape and the size of its watershed, the area of the terrain that contributes water to the depression, but also on other depressions filling up. Water that falls on the watershed of a full depression flows to a neighboring depression, effectively making its watershed larger and thus making it fill up faster. Modeling how depressions fill and spill into other depressions during a flash flood event is therefore an important problem.

The prior work on flood-risk analysis assumed that water only flowed along edges of the terrain, and further assumed a single-flow direction (SFD) model in which water flows from a point along one downward edge, say, the steepest descent one. ${ }^{1}$ In this article, we consider the multiflow direction (MFD) model in which water at a point may split and flow to multiple downslope neighbors which models flooding on the terrain more accurately. See Figure 1 for an example.

Flood-risk analysis under the MFD models raises many new challenges. Even when rain falls on a single point, as it flows across $\Sigma$, the water can split and reach many sinks, as illustrated in Figure 1. In turn, whenever a depression becomes full and starts spilling, this too can split and end up at many sinks across $\Sigma$. In contrast, under the SFD model, all water falling at a point flows to a single sink in $\Sigma$, and whenever a depression becomes full all the water spilling will again flow to another singular sink. As such, the terrain can be partitioned into "watershed" regions. Each region associated with one sink, corresponds to the set of all points on the terrain from where water flows to that sink. In the SFD model, water flows from a point to a sink only along a single path, while in the MFD model, water may reach from a point to the same sink along many paths. As such, flood queries on a terrain under the SFD model are answered by constructing a "flow tree" on the terrain, which is a tree with unweighted edges. In contrast, as we will see below, answering flood queries on a terrain under the MFD model requires constructing a weighted directed

\footnotetext{
${ }^{1}$ Since the modern data sets are high resolution and triangles are small, it is reasonable to assume that water flows along the edges.
} 
(acyclic) graph. Consequently, answering flooding queries under the MFD model is significantly more complicated and the approaches for the SFD model (e.g., as those in [3], [5], and [23]) do not extend to the MFD model.

In this article, we study three related problems; for each we assume we are given a terrain $\Sigma$, represented as a triangulated $x y$-monotone surface with $n$ vertices, and a rain distribution $\mathcal{R}$ describing the rate of rain over a region of $\Sigma$ :

- Terrain-Flood Query. Given a volume $\psi$ of rain falling according to $\mathcal{R}$, which vertices of $\Sigma$ will be flooded.

- Point-Flood Query. Given a volume $\psi$ of rain falling according to $\mathcal{R}$ and a point $q \in \Sigma$, determine whether $q$ is flooded.

- Flood-Time Query. Given a point $q \in \Sigma$, determine how much water (or equivalently how long) must fall before $q$ is flooded.

Our Results. We present three main results:

(i) We present an $O(n \log n)$-time algorithm to compute a terrain-flood query for a rain distribution $\mathcal{R}$ and volume $\psi$ (Section 4 ). We do so by traversing the terrain in a top-down manner and determining for each maximal depression whether it will get flooded. If a maximal depression is flooded, that extra water spills to its neighboring depression.

(ii) We first describe how the terrain-flooding algorithm can be expedited for answering pointflood queries. Although the worse-case running time remains $O(n \log n)$, it works faster in practice. Next, we present an $O(n \log n+n m)$-time algorithm for preprocessing $\Sigma$ into a data structure of size $O(\mathrm{~nm})$ for answering a point-flood query. Given a query point $q$, a rain distribution $\mathcal{R}$, and volume $\psi$, the data structure can answer a point-flood query in $O(|\mathcal{R}| k+$ $k^{2}$ ) time, where $k$ is the number of maximal depressions that contain the query point $q$ and $|\mathcal{R}|$ is the number of vertices with positive rainfall (Section 5). The data structure builds a directed acyclic graph that describes how tributaries of the point $q$ spill into downstream depressions, referred to as the tributary DAG of $q$.

(iii) We present an algorithm for answering a flood-time query. If the product of two $k \times k$ matrices can be computed in $O\left(k^{\omega}\right)$ time, it answers a query in $O\left(n k+k^{\omega}\right)$ (respectively, $O\left(|R| k+k^{\omega}+k^{2} \log n\right)$ ) time using $O(n)$ (respectively, $\left.O(n m)\right)$ space. We do so by showing how we can compute the flow spilling from many tributaries to a set of their downstream neighbors at once, utilizing fast matrix multiplication (Section 6).

We also give an $\alpha$-approximation algorithm, for any $\alpha>1$ for flood-time query that runs in $O\left(n \log n \log _{\alpha} \rho\right)\left(\right.$ respectively, $O\left(|\mathcal{R}| k+k^{2} \log \left(n \log _{\alpha} \rho\right)\right)$ time, using $O(n)$ (respectively, $\left.O(n m)\right)$ space where $\rho$ is a parameter depending on the terrain and point $q$ relating the ratio between the volumes of depressions on the terrain. The algorithm basically performs a binary search and uses the point-flood query data structure at each step.

We have implemented our algorithms for terrain-flooding, the point-flood query, and the approximate flood-time query and tested them on real terrains. We show that the algorithms are efficient in practice, across a variety of queries, and give some analysis as to the how varying the query affects the running time. We also compare terrain flood queries qualitatively under the MFD and SFD models, showing cases where the flooded regions differ significantly between the models (Section 7).

We note that this article is an expanded version of a paper that first appeared in the proceedings of the ACM SIGSPATIAL conference [19]. Here we give a new, faster algorithm for the terrainflood query, improving the run time complexity of queries from $O(n m)$ to $O(n \log n)$. We have also expanded the experimental section greatly. We implemented the new terrain-flooding algorithm, 
and also implemented the algorithm for answering point-flood queries and approximate flood-time queries.

\section{PRELIMINARIES}

We begin with a number of preliminary definitions, some of which closely follow Rav et al. [23].

Terrains. Let $\mathbb{M}$ be a triangulation of $\mathbb{R}^{2}$, and let $\mathbb{V}$ be the set of vertices of $\mathbb{M}$; set $n=|\mathbb{V}|$. We assume that $\mathbb{V}$ contains a vertex $v_{\infty}$ at infinity, and that each edge $\left\{u, v_{\infty}\right\}$ is a ray emanating from $u$; the triangles in $\mathbb{M}$ incident to $v_{\infty}$ are unbounded. Let $h: \mathbb{M} \rightarrow \mathbb{R}$ be a height function. We assume that the restriction of $h$ to each triangle of $\mathbb{M}$ is a linear map, that $h$ approaches $+\infty$ at $v_{\infty}$, and that the heights of all vertices are distinct. Given $\mathbb{M}$ and $h$, the graph of $h$, called a terrain and denoted by $\Sigma=(\mathbb{M}, h)$, is an $x y$-monotone triangulated surface whose triangulation is induced by $\mathbb{M}$.

Critical Vertices. There is a natural cyclic order on the neighbor vertices of a vertex $v$ of $\mathbb{M}$, and each such vertex is either an upslope or downslope neighbor. If $v$ has no downslope (respectively, upslope) neighbor, then $v$ is a minimum (respectively, maximum). We also refer to a minimum as a sink. If $v$ has four neighbors $w_{1}, w_{2}, w_{3}, w_{4}$ in clockwise order such that $\max \left(h\left(w_{1}\right), h\left(w_{3}\right)\right)<$ $h(v)<\min \left(h\left(w_{2}\right), h\left(w_{4}\right)\right)$, then $v$ is a saddle vertex.

Level Sets, Contours, Depressions. Given $\ell \in \mathbb{R}$, the $\ell$-sublevel set of $h$ is the set $h_{<\ell}=\left\{x \in \mathbb{R}^{2} \mid\right.$ $h(x)<\ell\}$, and the $\ell$-level set of $h$ is the set $h_{=\ell}=\left\{x \in \mathbb{R}^{2} \mid h(x)=\ell\right\}$. Each connected component of $h_{<\ell}$ is called a depression, and each connected component of $h_{=\ell}$ is called a contour. Note that a depression is not necessarily simply connected, as a maximum can cause a hole to appear.

For a point $x \in \mathbb{M}$, a depression $\beta_{x}$ of $h_{<\ell}$ is said to be delimited by the point $x$ if $x$ lies on the boundary of $\beta$, which implies that $h(x)=\ell$. A depression $\beta_{1}$ is maximal if every depression $\beta_{2} \supset \beta_{1}$ contains strictly more sinks than $\beta_{1}$. A maximal depression that contains exactly one sink is called an elementary depression. Note that each maximal depression is delimited by a saddle, and a saddle that delimits more than one maximal depression is called a negative saddle. For a maximal depression $\beta$, let $\operatorname{Sd}(\beta)$ denote the saddle delimiting $\beta$, and let $\operatorname{Sk}(\beta)$ denote the set of sinks in $\beta$. The volume of a depression $\beta$ of $h_{<\ell}$ is

$$
\operatorname{Vol}_{h}(\beta)=\int_{\beta}(\ell-h(v)) d v .
$$

Merge Tree. The maximal depressions of a terrain form a hierarchy that is easily represented using a rooted tree, called the merge tree $[8,16]$ and denoted by $\mathrm{T}_{h}$. Suppose we sweep a horizontal plane from $-\infty$ to $\infty$. As we vary $\ell$, the depressions in $h_{<\ell}$ vary continuously, but their structure changes only at sinks and negative saddles. If we increase $\ell$, then a new depression appears at a sink, and two depressions merge at a negative saddle. The merge tree is a tree that tracks these changes. Its leaves are the sinks of the terrain, and its internal nodes are the negative saddles. The edges of $\mathrm{T}_{h}$, the merge tree, are in one-to-one correspondence with the maximal depressions of $\Sigma_{h}$, that is, we associate each edge $(u, v)$ with the maximal depression delimited by $u$ and containing $v$. See Figure 2 for an example. We assume that $\mathrm{T}_{h}$ has an edge from the root of $\mathrm{T}_{h}$ extending to $+\infty$, corresponding to the depression that extends to $\infty$. For simplicity, we assume that $T_{h}$ is binary, that is, each negative saddle delimits exactly two depressions. Non-simple saddles can be unfolded into a number of simple saddles [12].

We extend the merge tree to the augmented merge tree by subdividing each edge $(u, v)$ of $\mathrm{T}_{h}$, into a sequence of edges $\left(u, e_{1}\right),\left(e_{1}, e_{2}\right), \ldots,\left(e_{k}, v\right)$. Each node $e_{i}$ in the augmented merge tree corresponds to a non-saddle vertex $w_{i} \in \sum$ such that $\operatorname{Sk}\left(\beta_{w_{i}}\right)=\operatorname{Sk}\left(\beta_{v}\right)$ (i.e., the depression delimited by $w_{i}$ contains the same set of minima as the depression delimited by the saddle $v$ ) and 


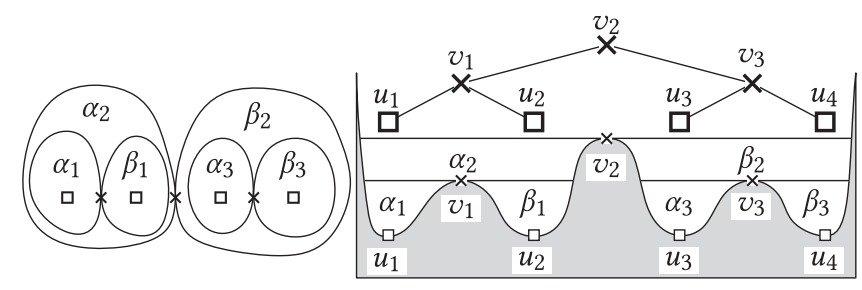

(a)

(b)

Fig. 2. Cross-section of an example terrain with saddle vertices $v_{1}-v_{3}$. Each saddle $v_{i}$ delimits two maximal depressions $\alpha_{i}$ and $\beta_{i}$. (a) Terrain seen from above. Sinks are marked with a square and saddles are marked with a cross. (b) Terrain seen from the side.

$h(u)>h\left(w_{1}\right)>h\left(w_{2}\right)>\cdots>h\left(w_{k}\right)>h(v)$. We sometimes refer to a vertex $w_{i}$ lying "on" the edge $(u, v)$ of the augmented T. We will see later that storing this additional information will be useful for computing flood-risk queries.

Let $u$ be a negative saddle, let $\left(u, v_{1}\right)$ and $\left(u, v_{2}\right)$ be two down edges in $T_{h}$ from $u$, and let $(w, u)$ be the up edge from $u$. We call the depression associated with $\left(u, v_{2}\right)$ (respectively, with $\left.(w, u)\right)$ as the sibling (respectively, parent) (depression) of that associated with $\left(u, v_{1}\right)$.

Any given point $q \in \mathbb{M}$ is contained in a sequence of maximal depressions $\alpha_{1} \supset \cdots \supset \alpha_{k} \ni q$, each $\alpha_{i}$ delimited by a saddle $v_{i}$ with sibling depression $\beta_{i}$. Note that these saddles form a path in $\mathrm{T}_{h}$ from $q$ to the root. We refer to the maximal depressions $\beta_{1}, \ldots, \beta_{k}$ as the tributaries of $q$ and denote them by $\mathcal{T}_{q}$. For a depression $\beta$ delimited by a point $q$, we will also use $\mathcal{T}_{\beta}$ to denote $\mathcal{T}_{q}$.

Van Kreveld et al. [16] gave an $O(n \log n)$-time algorithm for constructing the merge tree. The algorithm was later extended to 3D by Tarasov and Vyalyi [24], and to arbitrary dimensions by Carr et al. [8].

$\mathrm{T}_{h}$ can be preprocessed in $O(n)$ additional time so that for a point $x \in \mathbb{R}^{2}, \operatorname{Vol}\left(\beta_{x}\right)$, the volume of the depression delimited by $x$ can be computed in $O(\log n)$ time. In the following sections, we will be working with a fixed height function, so will drop the subscript $h$ from $\mathrm{T}_{h}, \mathrm{Vol}_{h}$, and the like.

\section{FLOODING MODEL}

Flow Graph and Flow Functions. We transform $\mathbb{M}$ into a directed acyclic graph $\mathcal{M}$, referred to as the flow graph, by directing each edge $\{u, v\}$ of $\mathbb{M}$ from $u$ to $v$ if $h(u)>h(v)$, and from $v$ to $u$ otherwise, i.e., each edge is directed in the downward direction. For each (directed) edge $(u, v)$, we define the local flow $\lambda(u, v)$ to be the portion of water arriving at $u$ that flows along the edge $(u, v)$ to $v$. If $\lambda(u, v)=0$, we can delete the edge $(u, v)$ from $\mathcal{M}$.

The value of $\lambda(u, v)$ is, in general, based on relative heights of the downslope neighbors of $u$. Note that the SFD model has $\lambda(u, v)>0$ for exactly one downslope neighbor of $u$. We will not focus on the specifics of the flow function used, and only assume that it is specified and for a pair $u, v$ can be retrieved in $O(1)$ time.

Following the edges of $\mathcal{M}$, water reaches a set of sinks of $\mathbb{M}$. We define a flow function $\phi: \mathbb{V}^{2} \rightarrow$ $[0,1]$, which specifies the proportion of water that flows from a vertex $u$ to another vertex $v$. Recall that unlike the SFD model, water can flow from $u$ to $v$ along many paths. The flow function is defined recursively as follows:

$$
\phi(u, v)=\left\{\begin{array}{cl}
1 & \text { if } u=v \\
\sum_{(u, w) \in \mathbb{E}(\mathcal{M})} \lambda(u, w) \cdot \phi(w, v) & \text { otherwise. }
\end{array}\right.
$$


For a maximal depression $\beta$, we define

$$
\phi(u, \beta)=\sum_{s \in \operatorname{Sk}(\beta)} \phi(u, s)
$$

to be the portion of water that reaches from a vertex $u$ to $\beta$. Recall that $\operatorname{Sk}(\beta)$ is the set of $\operatorname{sinks}$ in $\beta$.

Rain Distribution. We let $\mathcal{R}$ denote a rain distribution, which is specified as a probability distribution on the vertices of the terrain, that is, for each vertex $v \in \mathbb{V}, \mathcal{R}(v) \geq 0$ indicates the rate at which it rains on $v$, and we require that $\sum_{v} \mathcal{R}(v)=1$. We denote by $|\mathcal{R}|$ the number of vertices with positive rainfall in $\mathcal{R}$, and we assume that $\mathcal{R}$ is represented as a list of $|\mathcal{R}|$ pairs $(v, \mathcal{R}(v))$. In practice, $|\mathcal{R}| \ll n$.

Flooding Model. Our flooding model follows a similar depression filling model as Liu and Snoeyink [18]. When rain falls according to a distribution $\mathcal{R}$ on $\Sigma$, water flows according to the flow function and accumulates in depressions of $\Sigma$. When a maximal depression $\beta_{i}$ fills up, water spills from the simple saddle $v_{i}$ delimiting $\beta_{i}$ towards sinks in the adjacent depression delimited by the saddle. We refer to this event as a spill event.

The above process defines a sequence of spill events, each event marking a sink $u$ as full, and redistributing the rain falling on $u$ to other sinks. In our model, the maximal depressions of $\Sigma$ fill up at a constant rate between any two consecutive spill events. That is, after a spill event occurs at time $t_{1}$ and until the next occurs at time $t_{2}$, the volume of water in each elementary depression is a non-decreasing linear function of time.

Fill and Spill Rates. For a maximal depression $\beta$, we define the fill rateF $F_{\beta} \mathbb{R}_{\geq 0} \rightarrow[0,1]$ as the rate at which water is arriving in the depression $\beta$ as a function of time. That is, the rate at which rain is falling directly in $\beta$ plus the rate at which other depressions are spilling water into $\beta$. The fill rate $F_{\beta}$ is a monotonically nondecreasing piecewise-constant function. Similarly, we define the spill rate $S_{\beta}: \mathbb{R}_{\geq 0} \rightarrow[0,1]$ as the rate (as a function of time) at which water spills from $\beta$ through the saddle that delimits $\beta$. Let $\tau_{\beta}$, called the fill time, be the time at which $\beta$ becomes full. Let $\beta^{\prime}$ be the sibling depression of $\beta$. Then,

$$
S_{\beta}(t)= \begin{cases}0 & \text { if } t<\tau_{\beta} \vee \tau_{\beta^{\prime}} \leq \tau_{\beta}, \\ F_{\beta}(t) & \text { if } t>\tau_{\beta} \wedge \tau_{\beta^{\prime}}>\tau_{\beta} .\end{cases}
$$

By the definition of the fill rate, for any maximal depression $\beta$, its initial fill rate is

$$
F_{\beta}(0)=\sum_{s \in \operatorname{Sk}(\beta)} \sum_{v \in \mathbb{V}} R(v) \phi(v, s),
$$

which is how much rain water flows intially to the sinks of $\beta$. We can define $F_{\beta}(0)$ recursively using (2) as follows. Abusing the notation a little, let $F_{v}(0)$ denote the water reaching a vertex $v$ at time 0 . Then,

$$
\begin{gathered}
F_{v}(0)=R(v)+\sum_{(w, v) \in \mathbb{E}(\mathcal{M})} F_{w}(0) \lambda(w, v) \\
F_{\beta}(0)=\sum_{s \in \operatorname{Sk}(\beta)} F_{S}(0) .
\end{gathered}
$$

For any $t \geq 0$,

$$
F_{\beta}(t)=F_{\beta}(0)+\sum_{\alpha \in \mathcal{T}_{\beta}} S_{\alpha}(t) \phi(\operatorname{Sd}(\alpha), \beta) .
$$


That is, the fill rate of $\beta$ at time $t$ is the direct rain reaching $\beta$ plus the water spilling into $\beta$ from its tributaries that are full.

Fill and Spill Volumes. For a depression $\beta$, we similarly define $\mathcal{F}_{\beta}, \mathcal{S}_{\beta}: \mathbb{R}_{\geq 0} \rightarrow \mathbb{R}_{\geq 0}$ as fill- and spill-volume functions of $\beta$, i.e., $\mathcal{F}_{\beta}(t)$ tells how much water has arrived in depression $\beta$ by time $t$, and $\mathcal{S}_{\beta}(t)$ tells how much water has spilled from $\beta$ by time $t$.

$$
\mathcal{F}_{\beta}(t)=\int_{0}^{t} F_{\beta}(x) d x \text { and } \mathcal{S}_{\beta}(t)=\int_{0}^{t} S_{\beta}(x) d x .
$$

By definition of the spill rate,

$$
\mathcal{S}_{\beta}(t)=\max \left\{0, \mathcal{F}_{\beta}(t)-\operatorname{Vol}(\beta)\right\} .
$$

\section{TERRAIN-FLOOD QUERIES}

In this section, we describe an algorithm for answering a terrain-flood query. That is, given a rain distribution $\mathcal{R}$ and a volume $\psi$, determine which vertices of $\mathbb{M}$ will be flooded if a volume of $\psi$ rain falls according to the distribution $\mathcal{R}$. Recall that $\sum_{v \in \mathbb{M}} \mathcal{R}(v)=1$, so $\psi$ is the same as saying that rain falls for $\psi$ units of time on $\mathbb{M}$ with distribution $\mathcal{R}$.

We will first give a rough sketch of the algorithm. We assume the merge tree $\mathrm{T}$ is already computed. We sweep through the vertices in descending height order, maintaining two sets of values for each height $\ell$ :

(i) for each depression $\alpha_{i}$ in the sublevel set $h_{<\ell}$, maintain the fill volume $\mathcal{F}_{\alpha_{i}}(\psi)$ and

(ii) for each edge $(u, v)$ of the terrain where $h(v)<\ell \leq h(u)$, maintain the volume of rain flowing along which we will denote $\Lambda(u, v)$.

We note that for two depressions $\alpha, \beta$ that contain the same set of sinks, then $\mathcal{F}_{\alpha}(\psi)=\mathcal{F}_{\beta}(\psi)$. So these values need only be computed for each maximal depressions, which will be done when reaching a saddle vertex. At each vertex $v$, if $\mathcal{F}_{\beta_{v}}(\psi) \geq \operatorname{Vol}_{\beta_{v}}(\psi)$, we mark the depression as full and no longer need to process any vertices contained in $\beta_{v}$. If $\beta_{v}$ is not full, we instead compute how much water flows over $v$, as a sum of the rain falling directly on $v$ according to the distribution $\mathcal{R}$ plus the volume of rain flowing on $v$ for each downslope edge $(u, v)$. We then redistribute this water according to the flow function, and update the rain flowing across each edge $(v, w)$. Additionally, if $v$ is a saddle delimiting two maximal depressions $\alpha$ and $\beta$, we will compute how much water ends up in each depression (i.e., $\mathcal{F}(\alpha)$ and $\mathcal{F}(\beta)$ ). These values will be computed as a sum of the water falling directly on vertices in the depression, plus the water flowing on edges into the depression. If one depression, say $\alpha$ is full, we then determine how much excess water is spilling from $\alpha$ over $v$ into its sibling $\beta$ and redistribute this water on the downslope edges of $v$ into $\beta$.

Before describing the algorithm in detail, we give a few definitions. For a given depression $\beta$, let $\mathcal{R}(\beta)=\sum_{v \in \beta} \mathcal{R}(v)$ be the portion of rain falling directly in to $\beta$. Further, let $\boldsymbol{E}(\beta)=$ $\{(u, v) \mid u \notin \beta, v \in \beta\}$ be the set of edges that cross the contour delimiting $\beta$. We say these edges "cross into" $\beta$. Then, the volume of water in a depression $\beta$ is the amount falling directly in it, plus the amount of water flowing into it,

$$
\mathcal{F}_{\beta}(\psi)=\psi \mathcal{R}(\beta)+\sum_{e \in E(\beta)} \Lambda(e) .
$$

We will now show how to compute the terrain flood query utilizing these functions.

First, we compute how much rain directly falls in each maximal depression initially. For a maximal depression $\beta$, let $\hat{\mathcal{R}}(\beta)$ be the amount of rain falling on vertices in $\beta$ which are not contained 


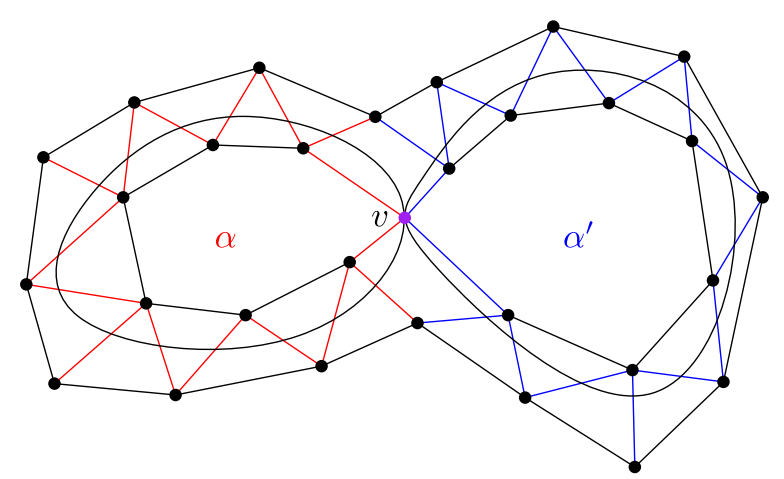

Fig. 3. Saddle $v$, marked in purple, delimits two maximal depressions $\alpha$ and $\alpha^{\prime}$. The edges in $E(\alpha)$ are marked in red, while those in $E\left(\alpha^{\prime}\right)$ are marked in blue.

in any other maximal depression. Iterating through each vertex $v$ with positive rainfall in $\mathcal{R}$, add a volume of $\mathcal{R}(v) \psi$ to the maximal depression containing $v$. Then, starting with the root of $\mathrm{T}$, we can recursively compute

$$
\mathcal{R}(\alpha)=\hat{\mathcal{R}}(\alpha)+\sum_{(\alpha, \beta) \in \mathrm{T}} \mathcal{R}(\beta)
$$

for all maximal depressions in T. This procedure takes $O(|\mathcal{R}|+m)$ time.

Next we process the vertices in descending height order. For a vertex $v$ with height $\ell$, let $\mathcal{D}_{\ell}=$ $\left\{\beta_{1}, \beta_{2}, \ldots, \beta_{k}\right\}$ be the set of non-full depressions corresponding to $\ell$-sublevel sets, i.e., $\mathcal{F}_{\beta_{i}}(\psi)<$ $\operatorname{Vol}\left(\beta_{i}\right)$. We will call these the active depressions. For each active depression $\beta_{i}$, we will compute $\mathcal{F}_{\beta_{i}}(\psi)$ and the values $\Lambda(u, v)$ for each $(u, v) \in E\left(\beta_{i}\right)$. One key point of the algorithm is that if two depressions $\alpha$ and $\beta$ contain the same set of sinks, it will be the case that $\mathcal{F}_{\alpha}(\psi)=\mathcal{F}_{\beta}(\psi)$, so we only need to explicitly compute these values at saddle vertices.

If $v$ is contained in an active depression, let $\beta_{v}$ be the depression delimited by $v$ and $\alpha_{v}$ be the smallest maximal depression containing $v$ (i.e., the maximal depression containing the same set of sinks as $\beta_{v}$ ). Then, as $\alpha_{v}$ contains $\beta_{v}$, the saddle delimiting $\alpha_{v}$ must be higher than $v$, which implies we have already computed $\mathcal{F}_{\alpha_{v}}(\psi)=\mathcal{F}_{\beta_{v}}(\psi)$. Using this value, we check if $\beta_{v}$ is full. If so, we mark all vertices contained in $\beta_{v}$ as flooded, and remove $\beta_{v}$ from the set of active depressions. Otherwise, for each edge $(v, w)$, we compute the water flowing along it as,

$$
\Lambda(v, w)=\lambda(v, w)\left(\mathcal{R}(v)+\sum_{(u, v) \in \mathbb{E}} \Lambda(u, v)\right) .
$$

Next, if $v$ is contained in an active depression and is a saddle vertex delimiting two maximal depressions $\alpha, \alpha^{\prime}$, we must also compute the volume of rain in each of the two depressions. To do so, partition the edges $E\left(\beta_{v}\right)$ into the two sets $E(\alpha)$ and $E\left(\alpha^{\prime}\right)$ and compute the volume of rain crossing into $\alpha$ (respectively, $\alpha^{\prime}$ ) as $\sum_{e \in E(\alpha)} \Lambda(e)$ (respectively, $\sum_{e \in E(\alpha)} \Lambda(e)$ ). See Figure 3 for an example. We claim that these sums can be computed in a total of $O(n \log n)$ time summed over all saddles, the details of which we will show later. Using this value along with the value of $\mathcal{R}(\alpha)$ (respectively, $\mathcal{R}\left(\alpha^{\prime}\right)$ ) in Equation (7), we can compute $\mathcal{F}_{\alpha}(\psi)$ (respectively, $\mathcal{F}_{\alpha^{\prime}}(\psi)$ ).

Finally, we use this value along with the depression volumes to check if $\alpha$ or $\alpha^{\prime}$ are flooded. Note that it will not be the case that both $\alpha$ and $\alpha^{\prime}$ are flooded, as this would mean that $v$ is flooded, and we would have already marked these depressions as flooded. If one is flooded, without loss of generality, let it be $\alpha$. In this case, mark $\alpha$ as flooded, and add $\alpha^{\prime}$ to $\mathcal{D}$. Then, the volume of rain spilling from $\alpha$ into $\alpha^{\prime}$ will be $\mathcal{F}_{\alpha}(\psi)-\operatorname{Vol}(\alpha)$. Let $\lambda^{\prime}(v, w)$ be the modified flow function, 

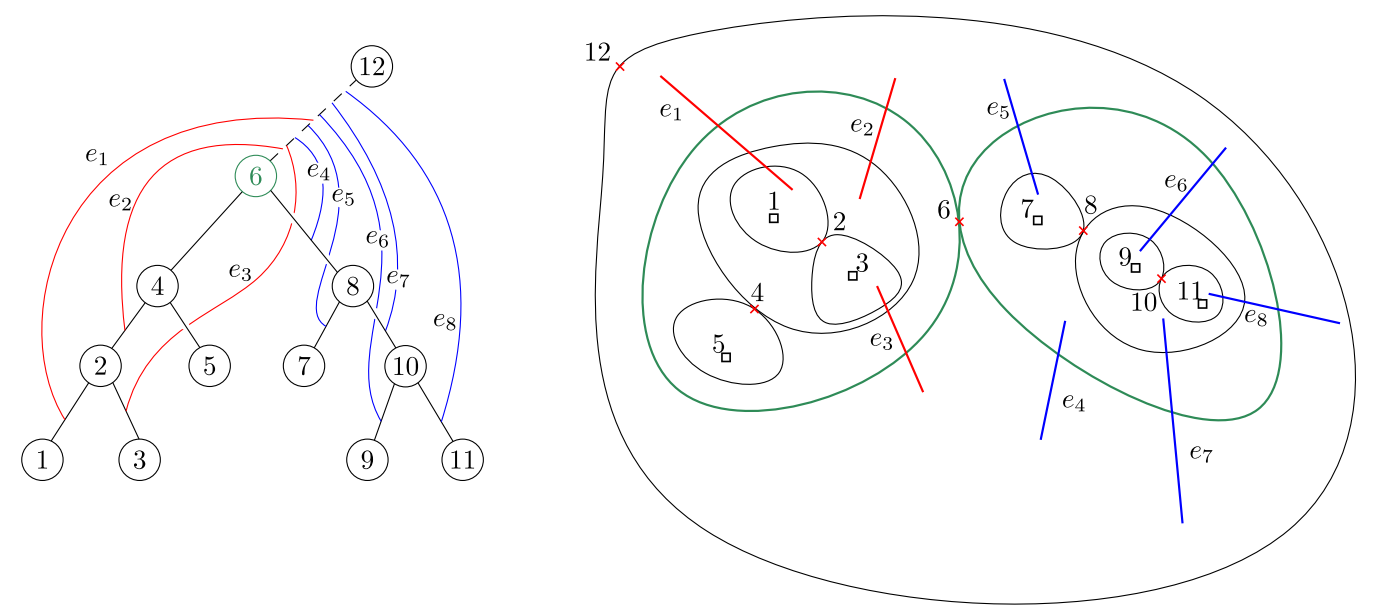

Fig. 4. Left: The augmented merge tree with the corresponding edges marked. Right: A terrain with contour lines drawn, with six saddle points drawn as squares, and eight edges $e_{1}-e_{8}$ marked. Edges take the index of the node below it in the augmented merge tree. For example, $e_{1}$ has index 1 , edge $e_{4}$ has index 8 , and edge $e_{7}$ has index 10 .

computed as if the flooded neighboring vertices have a height of $\ell$. Then, we update the flow of water along the edges from $v$ as follows:

$$
\Lambda(v, w)=\lambda^{\prime}(v, w)\left(\mathcal{R}(v)+\sum_{(u, v) \in \mathbb{E}} \Lambda(u, v)+\left(\mathcal{F}_{\alpha}(\psi)-\operatorname{Vol}(\alpha)\right)\right) .
$$

If neither $\alpha$ nor $\alpha^{\prime}$ are full, add them both to $\mathcal{D}$.

Partitioning Edges Efficiently. We will now show how to build a data structure to store the values of $\Lambda(u, v)$ so that, when we reach a saddle, we can partition the edges efficiently. First, compute the in-order traversal index for the nodes of T. Then, label each edge $(u, v)(h(u)>h(v))$ with the index of the node in T corresponding to the depression which contains the same set of sinks as $\beta_{v}$. In the extended merge tree, if $v$ is contained in the edge $(\alpha, \beta) \in \mathrm{T}$ with $h(\alpha)>h(\beta), v$ is associated with $\beta$. Our data structure will then store the values $\Lambda(u, v)$ in sorted order, keyed on this index. See Figure 4 for an example.

When we reach a saddle vertex $v$ delimiting two maximal depressions $\alpha, \alpha^{\prime}$ and wish to partition the edges, the edges with index smaller than the node associated with $v$ will cross into one depression, while those with index larger than $v$ will cross into the other. Noting that

$$
\sum_{e \in E\left(\beta_{v}\right)} \Lambda(e)=\sum_{e \in E(\alpha)} \Lambda(e)+\sum_{e \in E\left(\alpha^{\prime}\right)} \Lambda(e)
$$

then, as the value of the left-hand side, $\sum_{e \in E\left(\beta_{v}\right)} \Lambda(e)$, is known when we reach $v$, if we know the volume of water flowing into $\alpha$, we can determine the volume flowing in $\alpha^{\prime}$ in constant time, and vice versa. We can compute one of these values efficiently by taking partial sums of the first and last $k$ edges until we reach the dividing point associated with the saddle.

For example, consider partitioning the edges crossing into the two depressions delimited by the saddle labeled 6 in Figure 4. At this step in the algorithm, we would have the set of $E\left(\beta_{6}\right)$ as $e_{1}-e_{8}$ in sorted order along with the value $\sum_{i=1}^{8} \Lambda\left(e_{i}\right)$. Then, taking partial sums $\sum_{i=1}^{k} \Lambda\left(e_{i}\right)$ from the beginning of the sorted list (respectively, $\sum_{i=0}^{k-1} \Lambda\left(e_{8-i}\right)$ from the end) for $k=1,2,3$, we then find 
that $e_{3}$ is the last edge "to the left" of the saddle. We can then find the value of $\sum_{i=4}^{8} \Lambda\left(e_{i}\right)$ by taking the difference between the total sum and the partial sum of edges "to the left" of the saddle.

This takes time proportional to $O(\min \{|E(\alpha)|,|E(\beta)|\})$. Letting $|\alpha|$ denote the total number of edges with at least one vertex contained in $\alpha$ (noting $|\alpha| \geq|E(\alpha)|)$ and $T(\beta)$ be the total time to partition the edges for all saddles contained in $\beta$, we have that

$$
T\left(\beta_{v}\right)=O\left(\min \left\{|\alpha|,\left|\alpha^{\prime}\right|\right\}\right)+T(\alpha)+T\left(\alpha^{\prime}\right) .
$$

Noting that set of edges crossing into the two maximal depressions $\alpha, \alpha^{\prime}$ are disjoint, it follows that $|\alpha|+\left|\alpha^{\prime}\right| \leq\left|\beta_{v}\right|$. Using this, we can solve the recurrence, and see that $T\left(\beta_{v}\right)=O\left(\left|\beta_{v}\right| \log \left|\beta_{v}\right|\right)$.

Building the merge tree and precomputing how much rain falls in each maximal depression can be done in $O(n \log n)$ time. For each edge $e, \Lambda(e)$ can be computed in $O(1)$ amortized time, and we use an additional $O(\log n)$ time adding (and later removing) $\Lambda(e)$ from the sorted list. Finally, we spend a total of $O(n \log n)$ time at all saddles to partition the water flowing across edges. This gives an overall running time of $O(n \log n)$ to determine which vertices are flooded.

TheOREM 4.1. Given a triangulation of $\mathbb{M}$ of $\mathbb{R}^{2}$ with $n$ vertices, a height function $h: \mathbb{M} \rightarrow \mathbb{R}$ which is linear on each face of $\mathbb{M}$, a rain distribution $\mathcal{R}$ and a volume of rain $\psi$, the flooded vertices of $\mathbb{M}$ can be computed in $O(n \log n)$ time.

\section{POINT-FLOOD QUERY}

Given a rain distribution $\mathcal{R}$, a query point $q \in \mathbb{M}$, and a rain volume $\psi$, the point-flood query asks whether the point $q$ is flooded if a volume $\psi$ rain falls with distribution $\mathcal{R}$. Of course, the terrainflood query procedure described in Section 4 can answer this query, but our goal is to answer this query faster when possible. We exploit two observations: first, we need not compute the fill rate and fill time of all maximal depressions. In particular, suppose $q$ lies in a maximal depression $\beta$ and there are two children depressions $\beta_{1}$ and $\beta_{2}$ of the sibling depression $\beta^{\prime}$ of $\beta$. Then we need not compute the fill times of $\beta_{1}$ and $\beta_{2}$. It suffices to compute when $\beta^{\prime}$ fills and at what rate water spills from $\beta^{\prime}$ to the depression $\beta_{q}$. In fact, it suffices to consider the tributaries of $q$, defined in Section 2 . Second, we do not have to compute the fill rates of the tributaries of $\beta_{q}$ for all values time. Instead, it suffices to determine whether the volume of water in the depression $\beta_{q}$ is less than $\operatorname{Vol}\left(\beta_{q}\right)$.

We first define the notion of tributary graph that describes where the water spills when a tributary of $q$ fills. Next, we describe the functions that estimate how much water has arrived in a tributary of $q$ and how much it has spilled. Roughly speaking, we compute these estimates assuming that a tributary of $q$ fills before its sibling (which is a maximal depression containing $q$ ) fills. We prove that our estimates answer the point-flood query correctly. Finally, we describe a data structure which uses $O(m n)$ space and answers a query in $O\left(|\mathcal{R}| k+k^{2}\right)$ time.

Tributary Graph. For a point $q \in \mathbb{M}$, the tributary graph $\mathrm{G}[q]=\left(\mathrm{X}_{q}, \mathrm{E}_{q}\right)$ is a directed acyclic graph. $\mathrm{X}_{q}$ is the depression $\beta_{q}$ plus its tributaries, i.e., $\mathrm{X}_{q}=\mathcal{T}_{q} \cup\left\{\beta_{q}\right\}$. For a pair of depressions $\alpha, \beta \in \mathrm{X}$, we add the directed edge $(\alpha, \beta)$ to $\mathrm{E}_{q}$ if $h(\operatorname{Sd}(\alpha))>h(\operatorname{Sd}(\beta))$ (if $\beta=\beta_{q}$, then by $\operatorname{Sd}\left(\beta_{q}\right)$ we mean $q)$ and $\phi(\operatorname{Sd}(\alpha), \beta)>0$. We set the weights of the edge $(\alpha, \beta)$ to be

$$
w(\alpha, \beta)=\frac{\phi(\operatorname{Sd}(\alpha), \beta)}{\sum_{(\alpha, \gamma) \in \mathrm{E}_{q}} \phi(\operatorname{Sd}(\alpha), \gamma)},
$$

i.e., the weights are normalized so that the weighted out-degree of each node in $G[q]$ is 1 . See Figure 5 for an example.

It is expensive to compute $\mathcal{F}_{\beta}$ and $\mathcal{S}_{\beta}$ exactly for each tributary of $q$, so we define slightly different functions $\tilde{\mathcal{F}}_{\beta}, \tilde{\mathcal{S}}_{\beta}: \mathbb{R}_{\geq 0} \rightarrow \mathbb{R}_{\geq 0}$ for all $\beta \in \mathrm{X}_{q}$. They are fill, spill volume functions under 


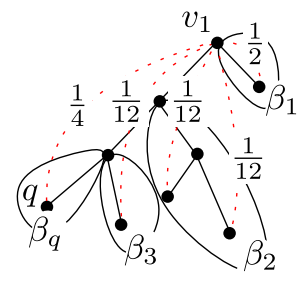

(a)

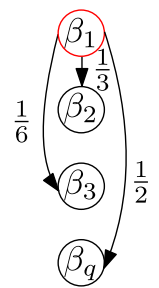

(b)

Fig. 5. (a) A merge tree $\mathrm{T}$, with tributaries $\left(\beta_{1}, \beta_{2}, \beta_{3}\right)$ of $q$ marked and flow $\phi\left(v_{1}, s\right)$ to each sink $s$ marked with dashed lines. (b) A subset of $\mathrm{G}[q]$, with the edge weights from $\beta_{1}$ marked.

the assumption that every tributary of $\beta_{q}$ fills before its sibling, that is, water spills from a tributary $\beta$ to various sinks in the sibling $\beta^{\prime}$ of $\beta$; note that $\beta^{\prime}$ is a depression containing $q$.

We define $\tilde{\mathcal{F}}_{\beta}, \tilde{\mathcal{S}}_{\beta}$ recursively using the tributary graph $\mathrm{G}[q]$ as follows:

$$
\begin{aligned}
& \tilde{\mathcal{F}}_{\beta}(t)=F_{\beta}(0) t+\sum_{(\alpha, \beta) \in \mathrm{E}_{q}} \tilde{\mathcal{S}}_{\alpha}(t) w(\alpha, \beta), \\
& \tilde{\mathcal{S}}_{\beta}(t)=\max \left\{0, \tilde{\mathcal{F}}_{\beta}(t)-\operatorname{Vol}(\beta)\right\} .
\end{aligned}
$$

The following lemma is the key observation.

LemmA 5.1. Let $\tau_{q}:=\tau_{\beta_{q}}$ be the fill time of the depression $\beta_{q}$. For any $\beta \in \mathrm{X}_{q}$,

(i) $\tilde{\mathcal{F}}_{\beta}, \tilde{\mathcal{S}}_{\beta}$ are monotonically non-decreasing functions.

(ii) For $t \leq \tau_{q}, \tilde{\mathcal{F}}_{\beta}(t)=\mathcal{F}_{\beta}(t)$ and $\tilde{\mathcal{S}}_{\beta}(t)=\mathcal{S}_{\beta}(t)$.

Proof. We begin by noting that, if the lemma holds for $\tilde{\mathcal{F}}_{\beta}$, for all $\beta \in \mathbf{X}_{q}$, it also holds for $\tilde{\mathcal{S}}_{\beta}$, so we prove the lemma for $\tilde{\mathcal{F}}_{\beta}$.

(i) We prove the claim by induction on the topological ordering of $\mathrm{X}_{q}$. For our base case, let $\alpha$ be the source of the tributary DAG, we have $\tilde{\mathcal{F}}_{\alpha}(t)=F_{\alpha}(0) t$, which is monotonically nondecreasing. By induction hypothesis, assume that $\tilde{\mathcal{F}}_{\alpha}(t)$ is monotonically nondecreasing, for all $\alpha$ appearing before $\beta$ in the topological ordering of $\mathrm{X}_{q}$. Then, $\tilde{\mathcal{F}}_{\beta}$ is the sum of monotonically nondecreasing functions, so it also is monotonically nondecreasing.

(ii) By the definition of $\tau_{q}$, the fill time of all maximal depressions containing $q$ is greater than $q$. Therefore, none of the sibling depressions of a tributary $\beta$ of $q$ (each of which is a maximal depression containing $q$ ) spills water into $\beta$, and therefore by definitions of $\mathcal{F}_{\beta}, \tilde{\mathcal{F}}_{\beta}, \mathcal{S}_{\beta}$, and $\tilde{\mathcal{S}}_{\beta}$, $\mathcal{F}_{\beta}(t)=\tilde{\mathcal{F}}_{\beta}(t)$ for all $t \leq \tau_{q}$.

Corollary 5.2. $\tilde{\mathcal{F}}_{\beta_{q}}(t)<\operatorname{Vol}\left(\beta_{q}\right)$ if and only if $t<\tau_{q}$.

Proof. If $\psi<\tau_{q}$, then $\tilde{\mathcal{F}}_{\beta_{q}}(t)=\mathcal{F}_{\beta_{q}}(t)<\operatorname{Vol}\left(\beta_{q}\right)$. The inequalities follows the definition of $\tau_{q}$. On the other hand, if $t \geq \tau_{q}$, then by (i) and (ii) of Lemma 5.1,

$$
\tilde{\mathcal{F}}_{\beta_{q}}(t) \geq \tilde{\mathcal{F}}_{\beta_{q}}\left(\tau_{q}\right)=\mathcal{F}_{\beta_{q}}\left(\tau_{q}\right)=\operatorname{Vol}\left(\beta_{q}\right) .
$$

By Corollary 5.2, given $\psi$, we compute $\tilde{\mathcal{F}}_{\beta_{q}}(\psi)$ and return yes if this quantity is at least $\operatorname{Vol}\left(\beta_{q}\right)$. We now describe the algorithm for computing $\tilde{\mathcal{F}}_{\beta_{q}}(\psi)$.

In the preprocessing step, we construct the merge tree $\mathrm{T}$ and preprocess it so that for a point $q \in \mathbb{M}$, (i) the edge of T containing $q$ and (ii) $\operatorname{Vol}\left(\beta_{q}\right)$ can be computed in $O(\log n)$ time. 
Additionally, for each vertex $v \in \mathbb{M}$ and for each maximal depression $\beta$, we store the value of $\phi(v, \beta)$. Recall that there are $O(m)$ maximal depressions, so we need $O(m n)$ storage. Actually, we need to store only non-zero values, in practice, the number of such pairs is much smaller than $m n$. The total time spent in computing this additional information is $O(\mathrm{~nm})$, by computing it for each sink in $O(n)$ time.

The preprocessing takes $O(n \log n+n m)$ time, and the size of the data structure is $O(n m)$.

For a query rain distribution $\mathcal{R}$ and a query point, we first find the edge $e$ of $\mathrm{T}$ containing $q$ and $\operatorname{Vol}\left(\beta_{q}\right)$. Given $e$, we compute the set $\mathcal{T}_{q}$ of tributaries of $q$ in $O(k)$ time by traversing $\mathrm{T}$ upward from $e$. We now construct the tributary graph $\mathrm{G}[q]=\left(\mathrm{X}_{q}, \mathrm{E}_{q}\right)$ in $O\left(k^{2}\right)$ time, using the precomputed values of $\phi(v, \beta)$ in (10).

To compute $\tilde{\mathcal{F}}_{q}(\psi)$ for all depressions $\beta \in \mathrm{X}_{q}$, we first compute $F_{\beta}(0)$, for each $\beta \in \mathrm{X}_{q}$, in $O(|\mathcal{R}|)$ time using the formula

$$
F_{\beta}(0)=\sum_{u: \mathcal{R}(u)>0} \mathcal{R}(u) \phi(u, \beta)
$$

Finally, we compute $\tilde{\mathcal{F}}_{\beta}(\psi)$ for every $\beta \in \mathrm{X}_{q}$, using the recurrence in a total of $\left|\mathrm{E}_{q}\right|=O\left(k^{2}\right)$ time. Hence, a point-flood query can be answered in $O\left(|\mathcal{R}| k+k^{2}\right)$ time. Putting everything together, we obtain the following:

Theorem 5.3. Given a triangulation of $\mathbb{M}$ of $\mathbb{R}^{2}$ with $n$ vertices, a height function $h: \mathbb{M} \rightarrow \mathbb{R}$, which is linear on each face of $\mathbb{M}$, a data structure of size $O(m n)$ can be constructed in $O(n \log n+m n)$ time so that a point-flood query can be answered in $O\left(|\mathcal{R}| k+k^{2}\right)$ time, where $|\mathcal{R}|$ is the complexity of the query rain distribution, $k$ is the number of maximal depressions containing the query point, and $m$ is the number of sinks in the terrain $(\mathbb{M}, h)$.

\section{FLOOD-TIME QUERIES}

In this section, we describe algorithms for answering flood-time queries: given a rain distribution $\mathcal{R}$ and a point $q \in \mathbb{M}$, how long rain must fall before $q$ becomes flooded. We first describe an exact algorithm for this problem and then an approximation algorithm. For both the exact and approximation algorithms, we assume that given the query point $q$, we have computed the (directed) tributary graph $\mathrm{G}[q]=\left(\mathrm{X}_{q}, \mathrm{E}_{q}\right)$, as described in Section 5 .

Exact Flood-Time Query. Roughly speaking, we wish to compute the fill rate $F_{\beta_{q}}$ of the depression $\beta_{q}$, which in turn requires computing the fill and spill rates of all tributaries of $q$. But computing them is expensive, so we use the same observation as in Section 5, namely, we compute "conditional" fill and spill rates of each $\beta \in \mathrm{X}_{q}$ under the assumption that every tributary of $\beta_{q}$ fills before its sibling. Abusing notation slightly, we use $F_{\beta}, S_{\beta}$ to denote these conditional fill and spill rates of $\beta$ for all $\beta \in \mathrm{X}_{q}$. By Lemma 5.1, $F_{\beta}, S_{\beta}$ are the exact fill rates for all $t \leq \tau_{q}$, so they determine the value of $\tau_{q}$ exactly. By definition,

$$
\begin{aligned}
& F_{\beta}(t)=F_{\beta}(0)+\sum_{(\alpha, \beta) \in \mathrm{E}_{q}} S_{\alpha}(t) w(\alpha, \beta), \\
& S_{\beta}(t)= \begin{cases}0 & \text { if } t<\tau_{\beta}, \\
F_{\beta}(t) & \text { if } t \geq \tau_{\beta} .\end{cases}
\end{aligned}
$$

Both $F_{\beta}$ and $S_{\beta}$ are piecewise-constant non-decreasing functions, and their values change only at fill times of tributaries of $\beta_{q}$. Using this observation, we can rewrite $F_{\beta}, S_{\beta}$ as follows: Let $\mathrm{E}_{q}=$ $\left\langle\beta_{1}, \beta_{2}, \ldots, \beta_{k+1}=\beta_{q}\right\rangle$ be the sequence of depressions sorted in descending order of their heights. For $1 \leq i \leq k+1$, we use $F_{i}, S_{i}, \tau_{i}$ to denote $F_{\beta_{i}}, S_{\beta_{i}}, \tau_{\beta_{i}}$, respectively, and for $j \leq i$, we set $w_{j, i}=$ $w\left(\beta_{j}, \beta_{i}\right)$. Recall that the fill rate of $\beta_{i}$ increases only when a $\beta_{j}$, for $j<i$, (i.e., $\beta_{j}$ is higher than 
$\beta_{i}$ ) fills and spills water into $\beta_{i}$. For a pair $i, j$, let $f_{i, j}$ (respectively, $s_{i, j}$ ) denote the increase in the fill rate $F_{i}$ (respectively, spill rate $S_{i}$ ) at time $\tau_{j}$. Note that $f_{i, j}, s_{i, j}=0$ for $j \geq i$. Set $f_{i, 0}=F_{i}(0)$ and $\tau_{0}=0$. Then,

$$
F_{i}(t)=\sum_{0 \leq j \leq i, \tau_{j} \leq t} f_{i, j} \quad \text { and } \quad S_{i}(t)=\sum_{1 \leq j \leq i, \tau_{j} \leq t} s_{i, j} .
$$

Furthermore,

$$
f_{i, j}=\sum_{j \leq \ell \leq i} w_{\ell, i} s_{\ell, j} \quad \text { for } 1 \leq j<i .
$$

That is, the increase in fill rate of $F_{\ell}$ is the increase in the spill rate of $\beta_{\ell}$ for $\ell<i$, at time $\tau_{j}$ times the proportion of water that spills from $\beta_{\ell}$ into $\beta_{i}$ summed over all tributaries higher than $\beta_{i}$. Note that the fill rate of $\beta_{\ell}$ does not increase at $\tau_{j}$ for $\ell<j$. Similarly, $s_{i, j}$ can be written as follows:

$$
s_{i, j}= \begin{cases}0 & \text { if } j>i \vee \tau_{j}<\tau_{i}, \\ \sum_{\ell<i, \tau_{\ell}<\tau_{i}} f_{i, j} & \text { if } j=i, \\ f_{i, j} & \text { if } j<i \wedge \tau_{j} \geq \tau_{i} .\end{cases}
$$

Let $\boldsymbol{F}=\left(f_{i, j}\right)_{\substack{1 \leq i \leq k+1 \\ 0 \leq j \leq k+1}}$ and $S=\left(s_{i, j}\right)_{1 \leq i, j \leq k+1}$ be fill and spill matrices; both of them are lower triangular matrices.

Let $F_{i}$ (respectively, $S_{i}$ ), for $1 \leq i \leq k+1$, denote the $i$ th row of $F$ (respectively, $S$ ). For a pair $1 \leq a, b \leq k+1$, let $\boldsymbol{F}_{a: b}$ (respectively, $S_{a: b}$ ) denote the submatrix of $\boldsymbol{F}$ (respectively, $S$ ) consisting of rows $a, a+1, \ldots, a+b-1 ; \boldsymbol{F}_{a}=\boldsymbol{F}_{a: 1}$ and $S_{a}=S_{a: 1}$.

After having computed $\boldsymbol{F}_{i}, S_{i}$ can be computed in $O(i)$ time using (13). We refer to this procedure as Spill_RAte $\left(i, \boldsymbol{F}_{i}\right)$. Here, we assume that we do not explicitly set $k-i+1$ entries of $S_{i}$ as 0 .

We now describe a recursive procedure for computing $\boldsymbol{F}$ and $S$, outlined in Figure 1. It takes as input two values $1 \leq a, b \leq k+1$ and a $b \times(k+1)$ lower-triangular matrix $\boldsymbol{F}_{a: b}^{<}=\left(f_{i j}^{a}\right)_{a \leq i<a+b}$, where $1 \leq j<i$

$$
f_{i, j}^{a}=\sum_{j \leq \ell<a} w_{\ell, i} s_{\ell, j}
$$

is a partial prefix sum of $f_{i, j}$ and the procedure returns $\boldsymbol{F}_{a: b}$ and $S_{a: b}$. We note that for $b=1$, $f_{a, j}=f_{a, j}^{a}$ for all $1 \leq j<a$. Hence, for $b=1$, given $\boldsymbol{F}_{a: 1}^{<}, \boldsymbol{F}_{a}$ can be returns in $O(a)$ time by simply setting $f_{a, 0}=F_{a}(0)$ and $f_{a, j}=f_{a, j}^{a}$ for $1 \leq j<a$. We call this procedure FilL_RATE $\left(a, \boldsymbol{F}_{a: 1}^{<}\right)$.

The only nontrivial step in FLOOD_TIME is the computation of matrix $\boldsymbol{B}$. A straightforward algorithm for computing $\boldsymbol{B}$ takes $O\left(b^{3}\right)$ time, but we can do better using a faster matrix multiplication algorithm, as follows:

Let

$$
W=\left(w_{\ell, i}\right)_{\substack{a \leq \ell<a+b / 2 \\ a+b / 2 \leq i<a+b}} \text { and } X=\left(s_{\ell, j}\right)_{\substack{a \leq \ell<a+b / 2 \\ 1 \leq j<a+b}}
$$

Then, $\boldsymbol{B}=\boldsymbol{W}^{T} \boldsymbol{X}$ (see Figure 6). Since $\boldsymbol{X}$ is not a square matrix, we partition $\boldsymbol{X}$ into $r=\left\lceil\frac{2 a}{b}\right\rceil$ blocks of $b / 2 \times b / 2$ matrices, i.e.,

$$
X=\left(X_{1}, \ldots, X_{r}\right) .
$$

Then $\left(\boldsymbol{W}^{T} \boldsymbol{X}_{1} \ldots \boldsymbol{W}^{T} \boldsymbol{X}_{r}\right)$ has all entries of $\boldsymbol{B}$ along with some 0 entries. We can keep the extra entries or discard them. Suppose two $n \times n$ matrices can be multiplied in $O\left(n^{\omega}\right)$ time, then $B$ can be computed in $O\left(r n^{\omega}\right)=O\left(\lceil a / b\rceil b^{\omega}\right)$ time. The rest of the steps in FlooD_Time take $O(b k)$ time. 


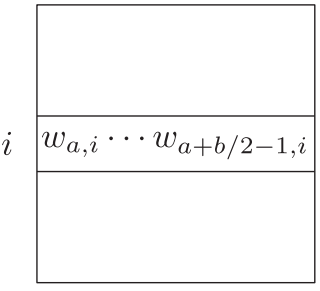

$W^{T}$

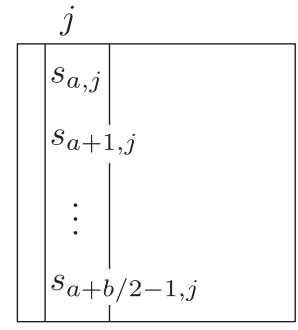

$X$

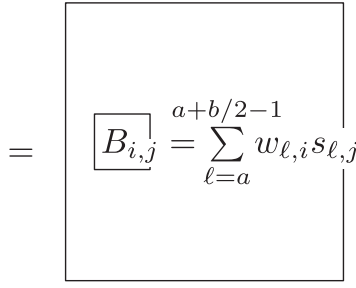

$B$

Fig. 6. An illustration of how each entry $\boldsymbol{B}_{i, j}$ is computed via matrix multiplication.

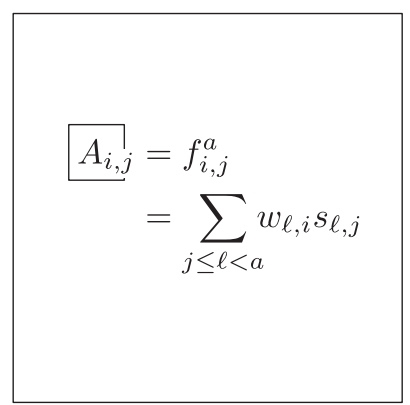

$A$

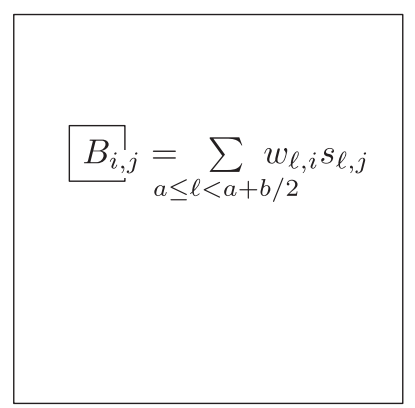

$B$

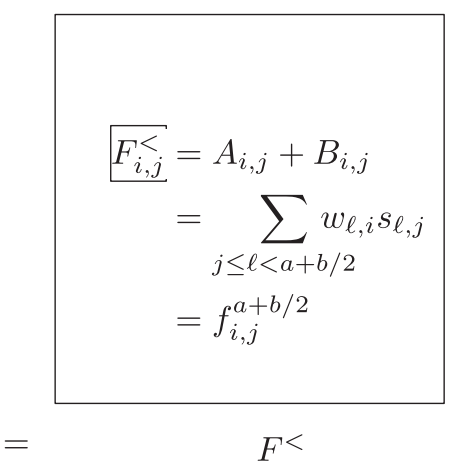

$F^{<}$

Fig. 7. After computing $B$, we then have $A+B=F^{<}$.

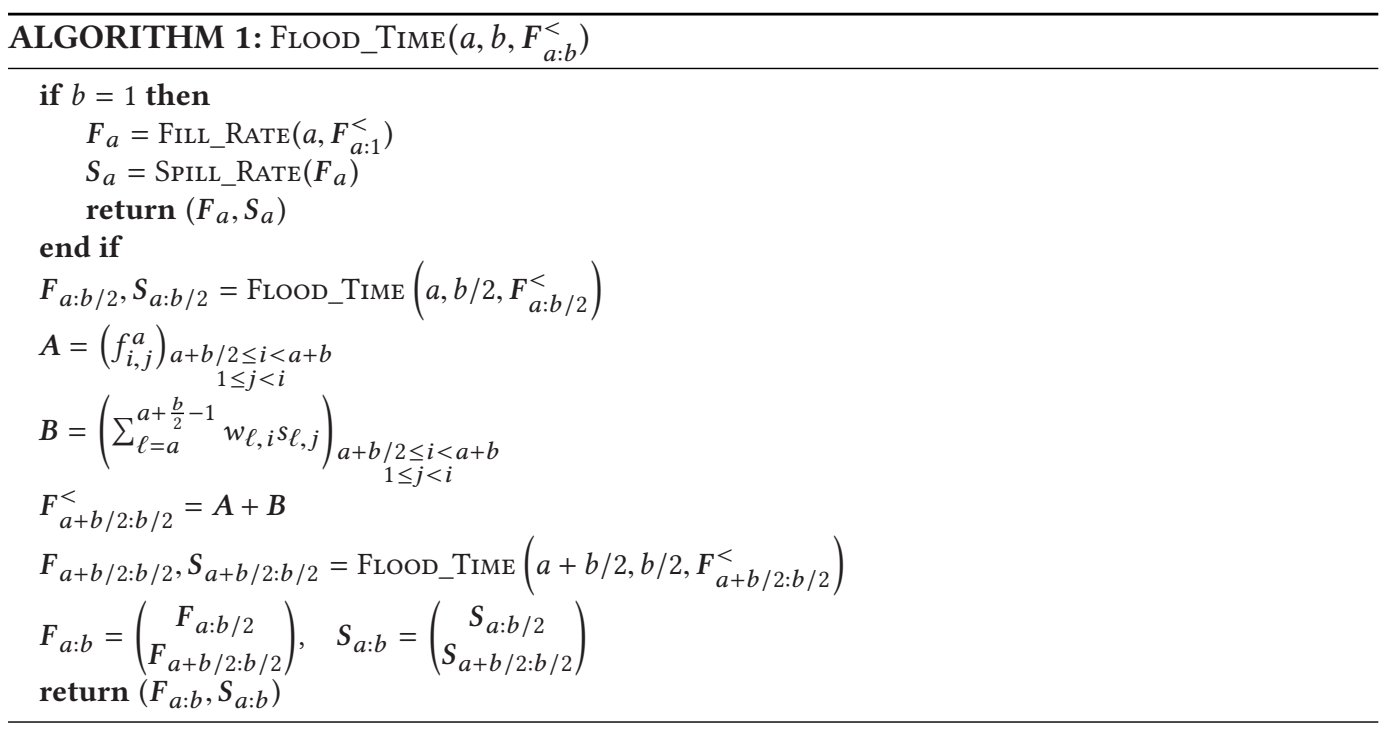

See Figure 7 for an example of how we compute $\boldsymbol{F}^{<}$. Let $T(a, b)$ denote the maximum running time of the procedure for FLOOD_Time $\left(a, b, F_{a: b}^{<}\right)$. Then, we obtain the following recurrence for $T(a, b)$ :

$$
T(a, b)= \begin{cases}O(a) & \text { for } b=1, \\ T\left(a, \frac{b}{2}\right)+T\left(a+\frac{b}{2}, \frac{b}{2}\right)+O\left(\left\lceil\frac{a}{b}\right\rceil b^{\omega}+b k\right) & \text { for } b>1 .\end{cases}
$$


The solution to the above recurrence is

$$
T(a, b)=O\left(\left[\frac{a}{b}\right\rceil b^{\omega}+b k \log k\right) .
$$

Since the procedure is initially called with $a=1$, and $b=k+1$, the overall running time of the recursive procedure is $O\left(k^{\omega}+k^{2} \log k\right)$. Combining this with the time spent in computing the tributary graph $\mathrm{G}[q]$, we obtain the following:

TheOREM 6.1. Given a triangulation of $\mathbb{M}$ of $\mathbb{R}^{2}$ with $n$ vertices and a height function $h: \mathbb{M} \rightarrow \mathbb{R}$ which is linear on each face of $\mathbb{M}$, a data structure of size $O(n)$ (respectively, $O(n m)$ ) can be constructed in time $O(n \log n)$ (respectively, $O(n m+n \log n))$ that, for a rain distribution $\mathcal{R}$ and a point $q$, can answer a flood-time query in time $O\left(n k+k^{\omega}+k^{2} \log k\right)\left(\right.$ respectively, $\left.O\left(|\mathcal{R}| k+k^{\omega}+k^{2} \log k\right)\right)$. Here $k$ is the number of maximal depressions containing $q, m$ is the number of sinks in the terrain $(\mathbb{M}, h)$, and $k^{\omega}$ is the time it takes to multiply two $k \times k$ matrices.

Approximate Flood-Time Query. Given a rain distribution $\mathcal{R}$ and point $q \in \mathbb{M}$, let the answer to the exact flood-time query be $\tau_{q}$. We say that an algorithm is an $\alpha$-approximation, for parameter $\alpha>1$, if it returns a value $\tau$ such that $\tau_{q} \leq \tau \leq \alpha \tau_{q}$.

Choosing indices $j_{i}$ such that

$$
\operatorname{Vol}\left(\beta_{j_{1}}\right) \leq \operatorname{Vol}\left(\beta_{j_{2}}\right) \leq \cdots \leq \operatorname{Vol}\left(\beta_{j_{k}}\right),
$$

let $V_{i}=\operatorname{Vol}\left(\beta_{j_{i}}\right)$ for ease of notation, and let $\operatorname{Vol}_{h+1}=h \operatorname{Vol}_{h}$. We note that $\operatorname{Vol}_{h+1} \geq \sum_{i=1}^{h} \operatorname{Vol}_{i}$, so after a volume of $\mathrm{Vol}_{h+1}$ rain falls, all depressions in $\mathrm{G}[q]$ will be full. It follows that $\sigma(\mathcal{R}, q) \leq$ $\mathrm{Vol}_{h+1}$. Performing a binary search, using $O(\log h)$ point-flood queries, we can compute the index $i$ such that $q$ is not flooded at time $V_{i}$, but $q$ is flooded at time $V_{i+1}$, in time $O\left(\left|\mathrm{E}_{q}\right| \log k\right)$.

Then, we perform a binary search on $\tau$ over the set of values $\left\{\alpha^{j} V_{i} \mid 0 \leq j \leq\left\lceil\log _{\alpha}\left(V_{i+1} / V_{i}\right)\right\rceil\right\}$ to find value $\tau$ such that

$$
\tau \leq \tau_{q} \leq \alpha \tau .
$$

We note that $\alpha \tau$ is an $\alpha$-approximation of the flood-time query.

Let $\rho=\max _{1 \leq j<h} \frac{\mathrm{Vol}_{j+1}}{\operatorname{Vol}_{j}}$, be the volume spread of $q$. We can get an $\alpha$-approximation of the fill time $\tau_{q}$ by performing $O\left(\log _{\alpha} \rho\right)$ point-flood queries. For example, if the largest ratio between two consecutively sized tributaries is at most $2^{h}$, then we can get a $2^{1 / h}$-approximation in time $O\left(\left|\mathrm{E}_{q}\right| \cdot \log h\right)$.

THEOREM 6.2. Given a triangulation of $\mathbb{M}$ of $\mathbb{R}^{2}$ with $n$ vertices and a height function $h: \mathbb{M} \rightarrow \mathbb{R}$ which is linear on each face of $\mathbb{M}$, a data structure of size $O(n)$ (respectively, $O(n m)$ ) can be constructed in time $O(n \log n)$ (respectively, $O(n \log n+n m))$ that for a rain distribution $\mathcal{R}$ and a point $q \in \mathbb{M}$, returns an $\alpha$-approximation to the flood-time query in time $O\left(n \log n \log _{\alpha} \rho\right)($ respectively, $O(|\mathcal{R}| k+$ $\left.\left.k^{2} \log \left(n \log _{\alpha} \rho\right)\right)\right)$, where $k$ is the number of maximal depressions containing $q$, and $\rho$ is the volume spread of $q$.

\section{EXPERIMENTS}

In this section, we present the experiments we have conducted on real terrains to demonstrate the efficiency of our algorithms and compare qualitatively the flooding under SFD and MFD models.

We have implemented the terrain-flood algorithm, described in Section 4, in C++, as well as a version of the point-flood and flood-time approximation algorithms, described below. We ran the experiments on a Dell R730 with 2 Intel Xenon ${ }^{\circledR}$, E5-2640 v4 2.4GHz 25M Cache and 256GB RAM running Linux. 


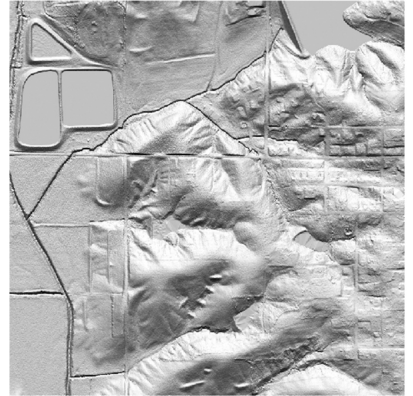

(a)

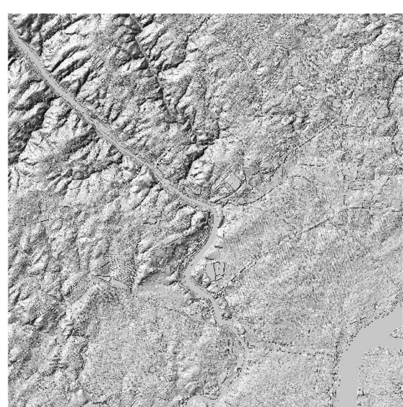

(b)

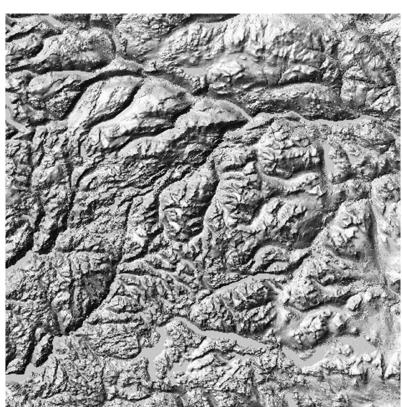

(c)

Fig. 8. A rendering of the three datasets used: (a) the Indiana dataset, (b) the Philadelphia dataset, and (c) the Norway dataset.

Datasets. We study the performance of our algorithm on three publicly available grid DEMs:

(i) The Indiana dataset (Figure $8(\mathrm{a})$ ), a $0.89 \mathrm{mi}^{2}$ model of an area $0.5 \mathrm{mi}$ northeast of Holland, Indiana, USA, extracted from the publicly available 5-ft resolution DEM of Indiana [14]. The dataset consists of $10^{6}$ grid vertices, and the terrain is relatively flat.

(ii) The Philadelphia dataset (Figure $8(\mathrm{~b})$ ), a $225 \mathrm{~km}^{2}$ model of an urban area in the northwest area of Philadelphia, extracted from the publicly available $3 \mathrm{~m}$ resolution DEM of Pennsylvania [21]. The dataset consists of $2.5 \times 10^{7}$ vertices, and we further consider subsets of this dataset of size $9 \mathrm{~km}^{2}, 81 \mathrm{~km}^{2}$, and $144 \mathrm{~km}^{2}$ consisting of $10^{6}, 9 \times 10^{6}, 1.6 \times 10^{7}$ points, respectively.

(iii) The Norway dataset (Figure $8(\mathrm{c})$ ), a $10,000 \mathrm{~km}^{2}$ model of a mountainous region located in the Jotunheimen National Park, Norway, exacted from the publicly available 10m resolution DEM of Norway [6]. The dataset consists of $10^{8}$ vertices, and we further consider subsets of this dataset of size $400 \mathrm{~km}^{2}$ and $2,500 \mathrm{~km}^{2}$ consisting of $4 \times 10^{6}$ and $2.5 \times 10^{7}$ points, respectively.

Flow Models. For the SFD model, for a pair of neighboring vertices $u, v$, we set

$$
\lambda(u, v)= \begin{cases}1 & \text { if } v \text { is lowest neighbor of } u \\ 0 & \text { otherwise. }\end{cases}
$$

For the MFD model, we use a flow model similar to Quinn et al. [22]. That is, let $\Delta(u, v)$ be the gradient of the edge $(u, v) \in \mathbb{M}$. We define the flow to be proportional to the gradient, with the total (local) flow out of every non-sink vertex being 1 :

$$
\lambda(u, v)= \begin{cases}0 & \text { if } u \text { is a sink, } \\ \frac{\max \{\Delta(u, v), 0\}}{\sum_{(u, v) \in \mathbb{E}} \max \{\Delta(u, v), 0\}} & \text { otherwise. }\end{cases}
$$

In Figure 1, we give an example of the two models on the Indiana dataset. The vertex marked $p$ is a local maximum, and each vertex $v$ with $\phi(p, v)>0$ is marked in blue, with darker blue indicating a larger value of $\phi(p, v)$. In Figure 1(a), under the SFD model flow is positive only along the path of steepest descent to a single sink. In Figure 1(b), we note that under the MFD model water falling at $p$ reaches multiple sinks.

Terrain-Flood Queries. We considered the rain distribution $\mathcal{R}$ to be rain falling: (i) on a single vertex, (ii) uniformly over a small rectangle, or (iii) uniformly over all vertices on the terrain.

Our experiments show that, when rain is falling at a single point, the areas that are flooded under the SFD and MFD models can be quite different. Under the MFD model some large areas 


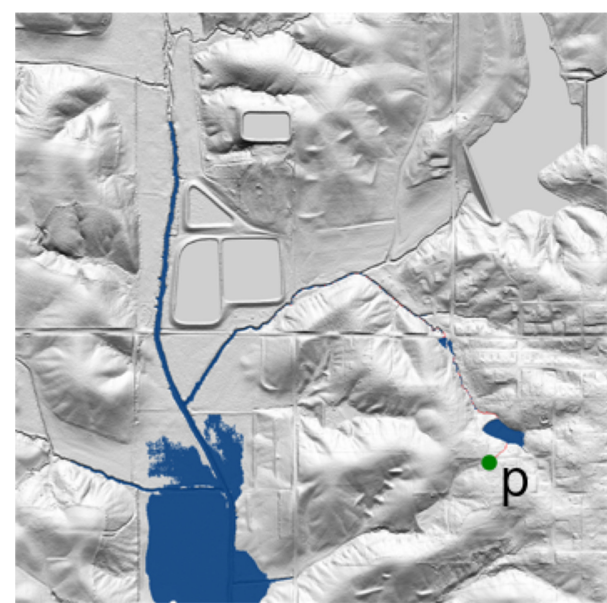

(a)

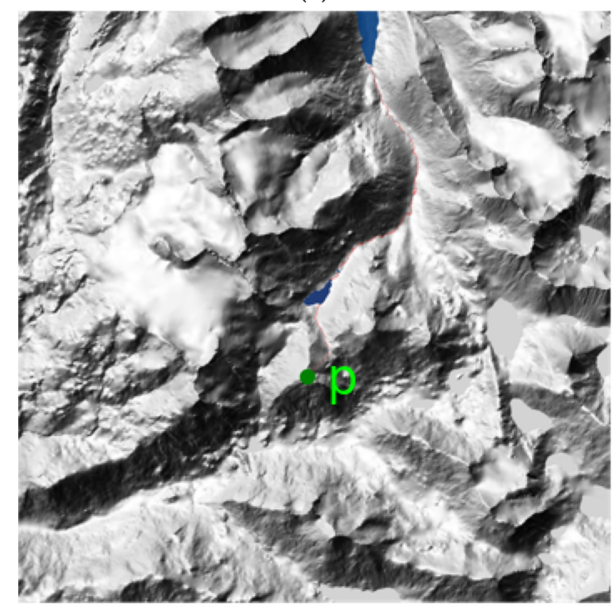

(c)

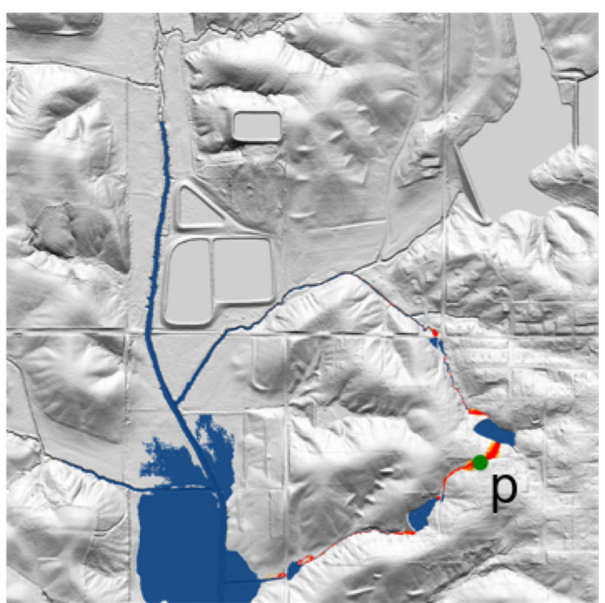

(b)

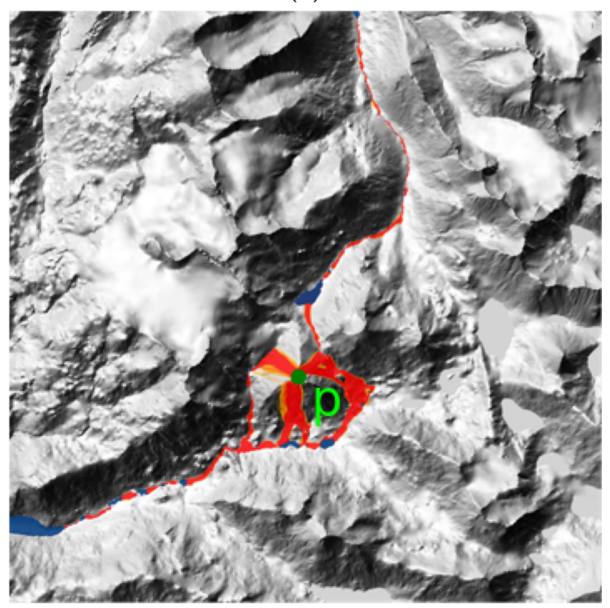

(d)

Fig. 9. $10^{5} \mathrm{~m}^{3}$ (respectively, $10^{7} \mathrm{~m}^{3}$ ) of rain falls at $p$ in the Indiana dataset in (b) and (a) (respectively, in the Norway dataset in (d) and (c)). The flooded regions of $\Sigma$ are marked in blue, with regions that water flows over marked in red. Water flows according to SFD in (a) and (c) and MFD in (b) and (d).

may become flooded that would not under the SFD model. As we increase the region on which rain is falling, we still see differences in the areas flooded, although they may be less pronounced. For example, the same general regions may be flooded, but under MFD more water might end up in one location as opposed to SFD, or water may reach more depressions. When we expand the rain distribution to be falling over the whole terrain, the regions which are flooded tend be very similar. Another unique difference in the two models, irrespective of the area of the region where rain is falling, is how water flows over the terrain. Under the SFD model, water flows along disjoint paths while under the MFD model it spreads more on the terrain (see, e.g., Figures 10-12.) We illustrate these observations below by a few examples.

For the case when rain falls at a single point, we computed the flooded areas with rain volume of $10^{5} \mathrm{~m}^{3}$ on the Indiana dataset and $10^{7} \mathrm{~m}^{3}$ on the Norway dataset. Figure 9 shows the terrain-flood query for two single-point rain distributions under both the SFD and MFD models. In Figure 9(a), we see that under the SFD model water follows a single path from $p$ north east, first filling a large 
region before spilling and filling a series of smaller regions as the water flows west towards a feature corresponding to a river. In Figure 9(b), under MFD, the water splits at $p$ and fills a number of depression to the south west of $p$. We see similar behavior in the Norway dataset in Figures 9(c) and 9(d). We additionally note that, under the MFD model in Figures 9(b) and 9(d), water spreads out more and flows across a wider path between full depressions.

For the case where rain is falling uniformly over a small square, we set the rain distribution to be uniform over a square of size $1 \mathrm{~km} \times 1 \mathrm{~km}$ and set $\psi=10^{6} \mathrm{~m}^{3}$ and computed the flooded area for the Norway dataset, under both SFD and MFD models. Figure 10 shows the queries along with enlarged images of the region on which it is raining and a region on the northern boundary which is flooded. We see that while similar areas become flooded, water spreads out across the peak more under the MFD model, and a larger fraction of the water flows to the southwest. In contrast, under the SFD model, the rain follows narrow bands outside of the rain region, and a larger fraction of the water flows to the north. In particular, the flooded region extends $0.35 \mathrm{~km}$ further towards the south.

For the case when rain falls uniformly over the entire terrain, we computed the flooded area when $1 \mathrm{~m}$ of rain falls uniformly on the Norway dataset with $2.5 \times 10^{7}$ vertices, and when $1 \mathrm{~m}$ of rain falls uniformly on the Philadelphia dataset. Figure 11 shows the flood area under both models. Overall, the terrain flood queries look similar under the SFD and MFD models; however, we note, under the SFD model, water flows across narrow regions corresponding to the path of steepest descent, while under the MFD model, water tends to spread out and flow across wider paths. Figures 12(a) and 12(b) show a smaller region of the Norway terrain in more detail. We note that, under the SFD model, water flows along many disjoint paths, while under the MFD model, the water spreads out and accumulates more along major valleys of the terrain. Figures 12(c) and 12(d) shows a smaller region of the Philadelphia terrain in more detail. We note that under the MFD model, the regions over which the most water flows clearly correspond to streets in Philadelphia. For example, the line passing diagonally from the northwest to the southeast corresponds to Ridge Avenue.

Terrain-Flood Performance. Building the merge tree and preprocessing it to compute the depression volume of any point as well as to perform lowest common ancestor (LCA) queries took an average of 1.33 seconds over five trials for the Indiana dataset.

We also ran tests over the Philadelphia dataset, taking subregions with $1.6 \times 10^{7}, 9 \times 10^{6}$, and $1 \times 10^{6}$ vertices, and on the Norway dataset. Figure 13 shows the preprocessing time as the number of vertices in the terrain was increased. We see that, in practice, the preprocessing time is near linear in the size of the terrain. While preprocessing does require sorting the nodes, which takes $O(n \log n)$ time, in practice the constant on this term is much smaller than that of the linear steps in the preprocessing.

Figure 14 gives a summary of the running time of the terrain flood query on the Indiana dataset as we change the number of points with positive rain in $\mathcal{R}$ and the amount of rain $\psi$. In Figure 14(a), we fix $\mathcal{R}$ to be uniform rain distribution over a fixed vertex and vary $\psi$, the volume of rain. In Figure 14(b), the distribution $\mathcal{R}$ is uniform over a square with a fixed upper left corner but varying its side length from 1 to 40 vertices; with the total rain volume fixed. For both these tests, we chose 500 vertices uniformly at random in both the Indiana dataset.

In Figure 14(a), we see that as the volume of rain increases the running time first increases until it reaches a peak and then decreases, becoming very fast with the largest volumes of rain. When a small amount of rain is falling in a small area, water reaches very few depressions and thus only a small portion of the merge tree is explored by the algorithm. As the volume and area of rain increases, the algorithm explores larger portions of the merge tree leading to an increase in the 


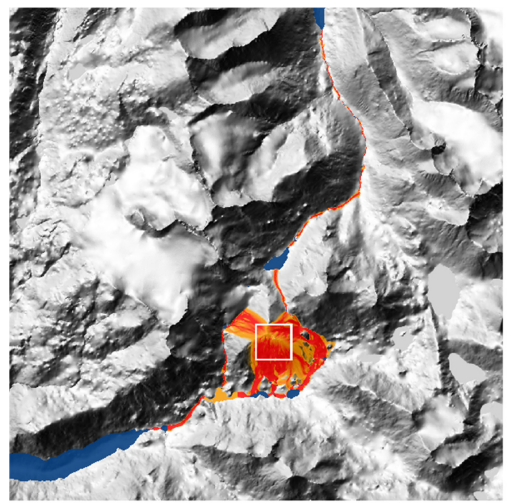

(a)

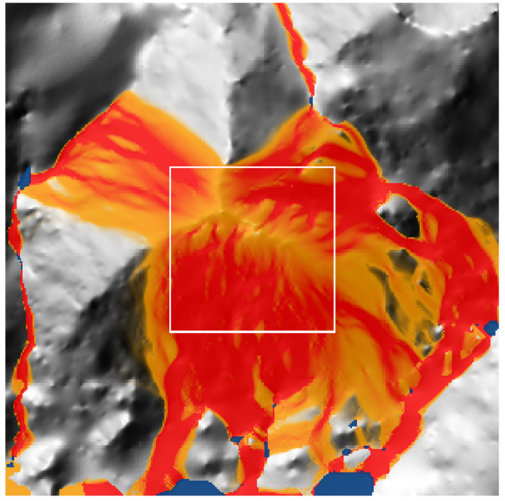

(c)

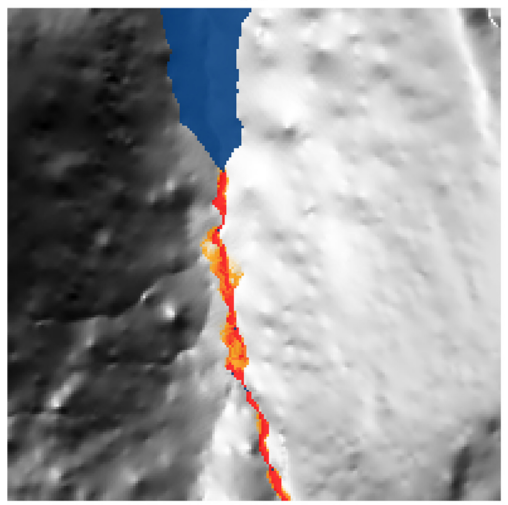

(e)

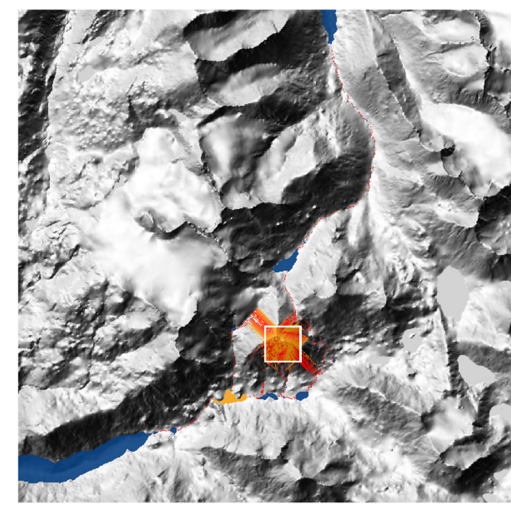

(b)

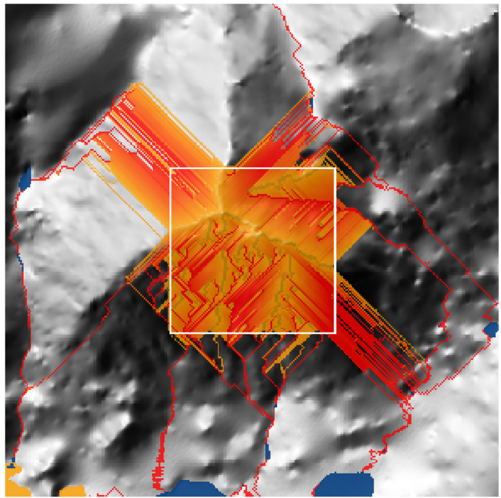

(d)

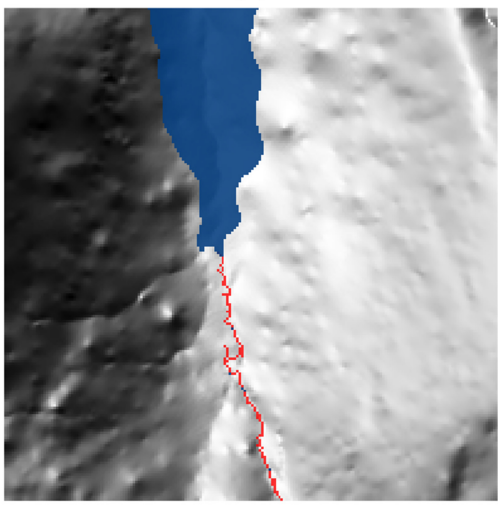

(f)

Fig. 10. $100 \mathrm{~m}$ of rain falls uniformly over the rectangle outlined in white in the Norway dataset: (a) water flows according to MFD, (b) water flows according to SFD, (c) and (d) show a $3 \mathrm{~km} \times 3 \mathrm{~km}$ area around the region it is raining on, (e) and (f) show a $2 \mathrm{~km} \times 2 \mathrm{~km}$ area on the northern boundary which is flooded. 


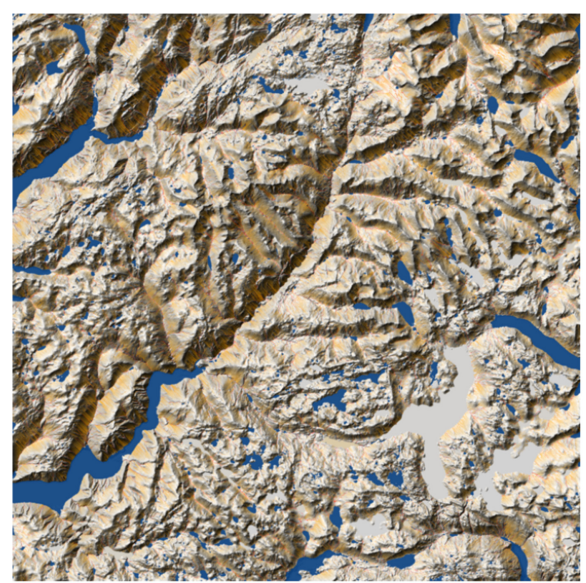

(a)

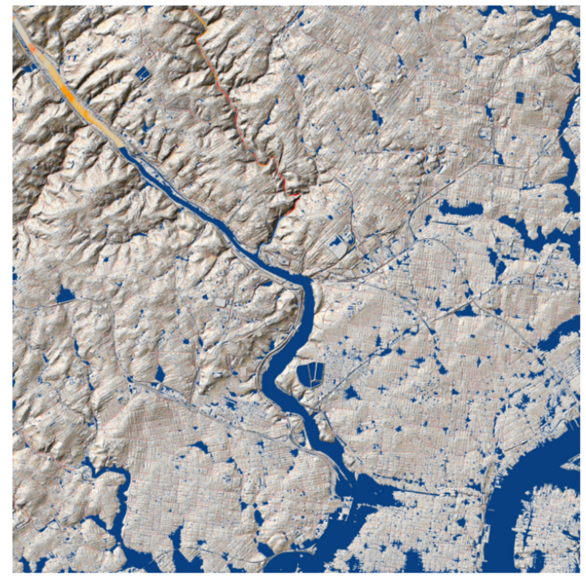

(c)

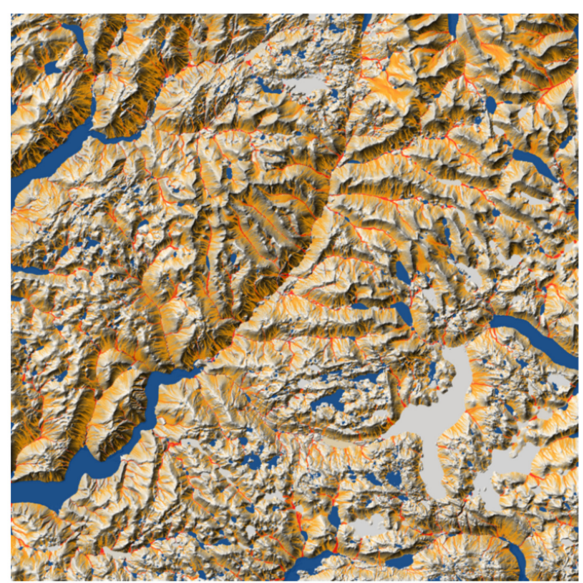

(b)

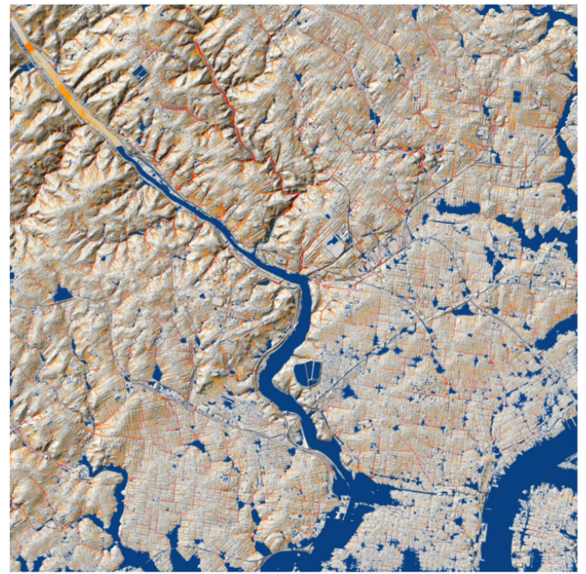

(d)

Fig. 11. $1 \mathrm{~m}$ of rain falls uniformly over the Norway dataset with $2.5 \cdot 10^{7}$ vertices in (a) and (b) (respectively, over the Philadelphia dataset in (c) and (d)). The flooded regions of $\Sigma$ are marked in blue, with regions that water flows over marked in red. Water flows according to SFD in (a) and (c) and MFD in (b) and (d).

running time. However, once the volume of rain increases further, large depressions get filled and the algorithm succeeds in pruning large portions of the merge tree. Roughly speaking, the running time of the algorithm is proportional to the number of depressions that are partially filled.

In Figure 14(b), we see that, while the running time increases with the number of vertices with positive rainfall, it grows slower than the linear dependence indicated by the worst-case time analysis. The query is somewhat faster when there is positive rainfall on a single point, this is probably in part due to optimizations in the code for initializing rain on the merge tree in the case when rain falls on a single point. We saw similar behavior for queries of varying area on the Philadelphia dataset.

Point-Flood Queries. As noted in Section 5, the terrain flood query can be used to perform pointflood queries in $O(n \log n)$ time using a linear-size data structure. We implemented a modified version of the terrain flood query, which has the same worst case complexity, but is designed to have faster practical running time. We do so by only considering vertices which delimit depressions 


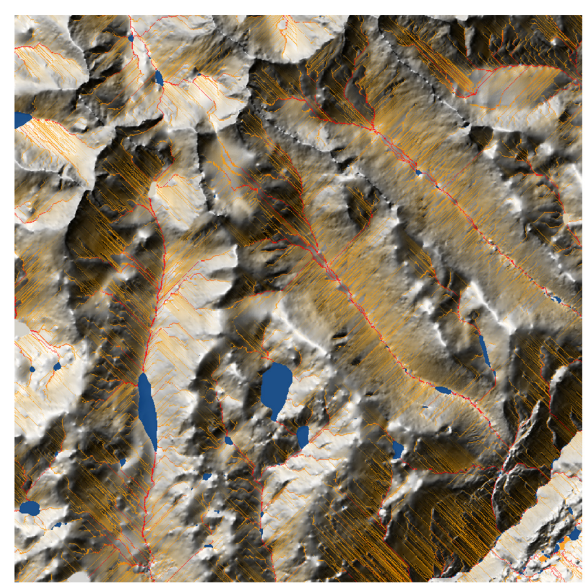

(a)

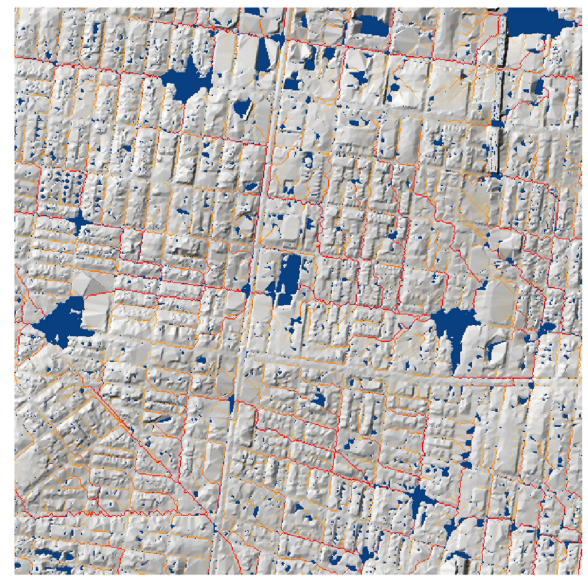

(c)

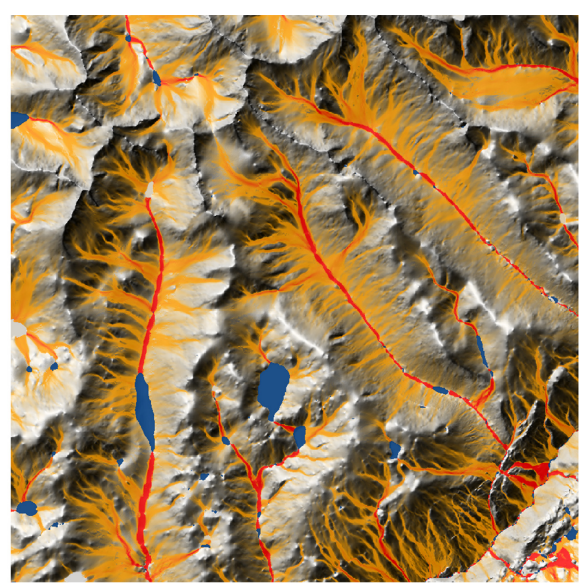

(b)

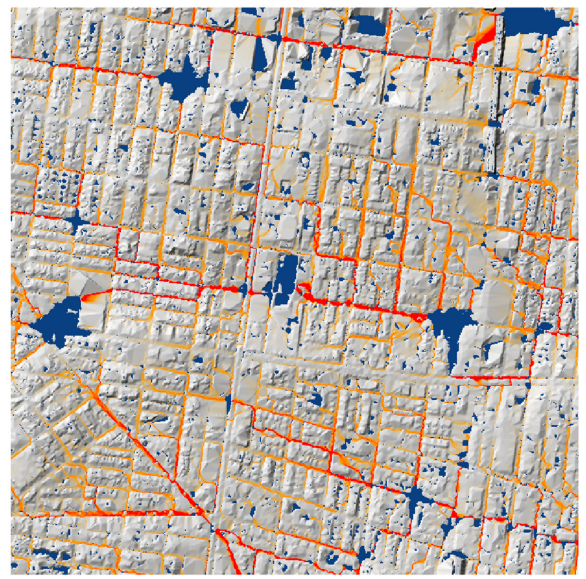

(d)

Fig. 12. $1 \mathrm{~m}$ of rain falls uniformly over the Norway dataset with $2.5 \cdot 10^{7}$ in (a) and (b) (respectively, over the Philadelphia dataset in (c)-(d)). These figures show a small region of the terrains enlarged. The flooded regions of $\Sigma$ are marked in blue, with regions that water flows over marked in red. Water flows according to SFD in (a) and (c) and MFD in (b) and (d).

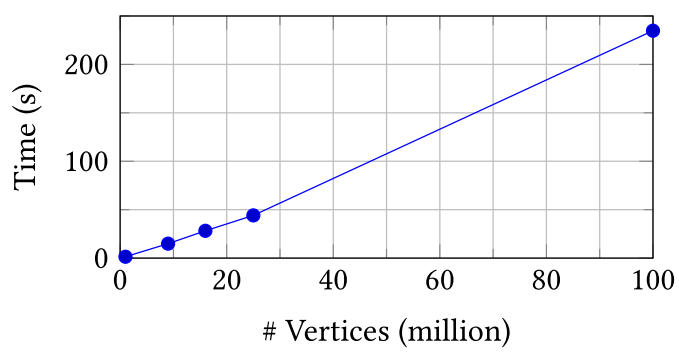

Fig. 13. Preprocessing time for the Philadelphia and Norway datasets as we vary the input size of the terrain. 


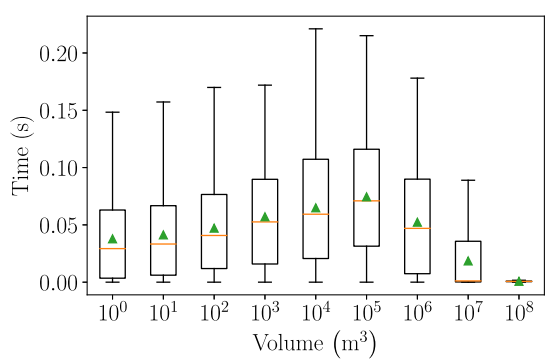

(a)

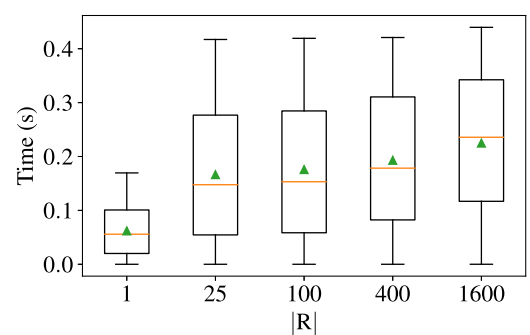

(b)

Fig. 14. On the Indiana dataset, (a) 500 terrain flood queries were performed for each of nine varying volumes of rain and (b) 500 terrain flood queries were performed for each of five varying rain region sizes. The results are summarized as box plots without outliers. Each box spans the first through third quartile of the query times, with the median marked with a line. The average query time is marked with a green triangle.

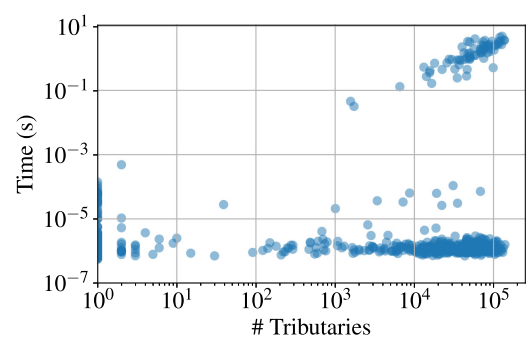

(a)

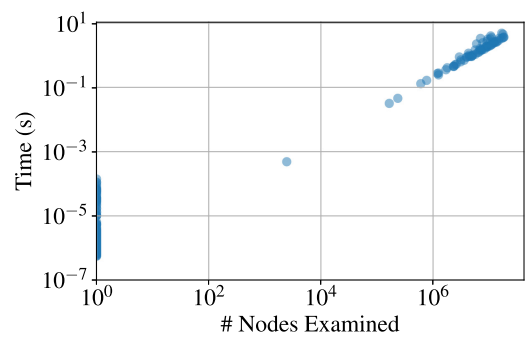

(b)

Fig. 15. One thousand point-flood queries were performed on the Philadelphia dataset. (a) Each point-flood query time is plotted against the number of tributaries of the query point up to the tributary containing the point on which rain is falling. (b) Each point-flood query time is plotted against the number of nodes of the extended merge tree examined by the algorithm.

containing the query point $q$. For saddle vertices, we only consider those which delimit a tributary of $q$. At these points, we either find that the tributary is full, and add the water spilling towards $q$, or find that it is not full, in which case we need not look at the vertices in the tributary as they will have no effect on whether $q$ is flooded or not. Similarly, for non-saddle vertices, if the depression delimited by the vertex does not contain $q$, water from this vertex cannot reach $q$, so we ignore it.

We considered 1,000 point-flood queries on the Philadelphia dataset, with $9 \times 10^{6} \mathrm{~m}^{3}$ of rain falling on a single vertex, chosen uniformly at random, and a query point, also chosen uniformly at random.

Figure 15(a) shows the query time compared to the number of tributaries of the query point up to the tributary which contains the point on which it is raining. We see many of these point-flood query times are extremely fast. In the case where there are no such tributaries, that is when the rain is falling within the depression delimited by the query point, we need only compare the volume of rain to the volume of this depression. On the other hand, even when there are a large number of tributaries, if the rain volume is less than the volume of the tributary into which it is raining, we know the query point will not be flooded. For the point-flood queries which took relatively longer, we see a linear correlation between the number of tributaries and the query time. 


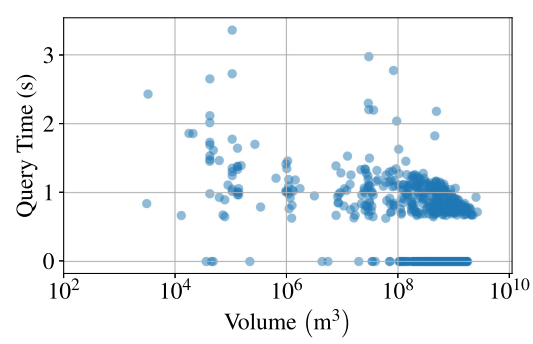

(a)

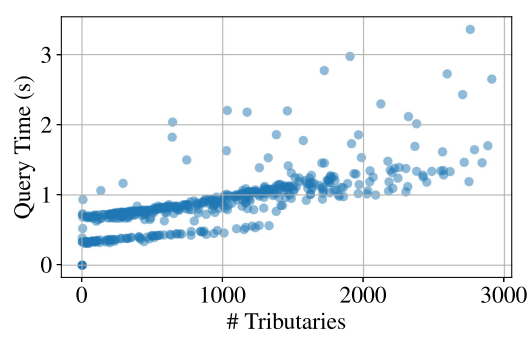

(b)

Fig. 16. One thousand approximate flood-time queries were performed on the Philadelphia dataset with $10^{6}$ vertices, with approximation factor $\alpha=2$. The query time is plotted against (a) the resulting approximate flood-time (given as volume) and (b) the number of tributaries of the query point.

Figure 15(b) shows the query time compared to the number of nodes in the extended merge tree examined by the algorithm. Many queries only require looking at a single node and these are therefore very fast. For queries that require looking at more nodes, the running time is strongly correlated with the number of nodes examined.

Flood-Time Approximation Queries. As noted in Section 6, the flood-time query can be approximated by finding an upper and lower bound on the flood-time, and using the point-flood query to perform a binary search. Utilizing the point-flood query described above, we implemented a version of the flood-time approximation. As noted earlier, by considering a fixed rate of rain, the flood-time query can be thought of as a flood-volume query. With that in mind, we will refer to the results of the queries in terms of total volume rather than time to avoid confusion between the time it took to perform the query and the result thereof.

We considered 1,000 flood-time queries on the Philadelphia dataset with $10^{6}$ points, with rain falling on a single vertex, chosen uniformly at random and a query point also chosen uniformly at random. We then calculated a 2-approximation of the volume of rain needed to fall at the chosen point before the query point was flooded.

Figure 16(a) shows the time of these queries compared to the approximate flood-volume. Figure 16(b) shows the time of these queries compared to the number of tributaries between the rain and the query point. As the number of tributaries increases, there is a slight increase in the query time, corresponding to the fact that we first perform the binary search on the volumes of these tributaries. We also note that when the depression delimiting the query point contains the point on which it is raining, we know the exact flood-volume will be equal to the volume of this depression, so the query time is extremely fast. There is no strong correlation between the floodvolume and query time, with the exception of a slight downward trend at the upper extreme of the volume. This corresponds to a negative correlation between the flood volume and number of tributaries, as query points corresponding to vertices higher in the extended merge tree tend to have larger volumes and fewer tributaries.

\section{RELATED WORK}

Flood-risk analysis is an important problem. It has been widely studied in multiple research communities, and many different approaches have been taken to address this problem. One such approach, coming from the hydrology community, focuses on accurately simulating fluid dynamics, using non-linear partial differential equations such as the Navier-Stokes equations. These equations have no closed form solutions, and are usually solved using numerical methods. They often account for additional factors, such as the effects of different terrain types, and drainage networks. 
While these models tend to be the most accurate, naive applications are computationally expensive. As such, there have been many approaches to reducing the computational cost of these methods.

Bates and De Roo [7] developed a model for simulating flooding on digital elevation models (DEMs) using two sets of equations, one to model flow within channels and one to model flow over floodplains. Comparing it to prior flood events, they found it correctly predicted $81.9 \%$ of (non)-inundated areas in the terrain. Volp et al. [26] developed a model that attempts to lower the computational cost of modeling by computing the flow on a coarse grid, while utilizing a finer subgrid to account for the roughness of the terrain. They show that this model can accurately approximate the behavior of a finer grid while being 50-100 times faster, as well as showing that the inclusion of the subgrid improves the approximation. Ghimire et al. [13] approximate flood equations on urban areas using a cellular automata model. This defines a set of rules for updating the water at each point in the terrain based on the state of its neighbors. They found this model produced similar results to an existing hydrological model, while improving computation time by a factor of 30 .

Recently, machine-learning-based approaches have been proposed for predicting flood risk. These approaches are relatively fast, while maintaining a reasonable level of predictive power. Tehrany et al. [25] tested the efficacy of various support vector machine (SVM) kernels at predicting the overall flood hazard of points in a terrain, that is the probability that a point would be flooded in a rain event. Their dataset consisted of a historical flood event in Malaysia and they considered a number of features for each point in the terrain such as slope, altitude, surface runoff and distance from a river. Chang et al. [10] used self-organizing maps and neural networks to forecast the flood inundation in the near future (1-3 hours) given the current inundated areas. They trained and tested their model using historical data of a region of Taiwan that has frequent floods, dividing the region into smaller parts and building a model for each subregion.

Finally, there has also been extensive work on modeling water flow on a terrain in the GIS community $[1-5,9,11,15,18,20,22,23]$. These approaches use simpler models, focusing on the geometry of the terrain. These tend to be more computationally efficient and suitable for large datasets. However, the simplifying assumptions mean that they may not be as accurate as PDEbased models in all situations. For example, they do not take into account absorption of water into the ground, and are thus more suitable for flash floods wherein most of the flooding occurs over a shorter timespan.

Some of these models first remove all depressions by flooding the terrain, that is, they conceptually pour water onto the terrain until all depressions are filled [2, 15, 20]. However, this approach often leads to unrealistic flow networks, since many important geographical features are removed. Therefore, several methods based on partial flooding algorithms that flood only "small" depressions (based on height, volume, or area) have been proposed [1, 4, 9, 11]. Partial flooding methods provide a basis only for approximate solutions to flow modeling, as the underlying assumption is that all "small" depressions are flooded at a certain time, while all "big" depressions are not. To model the flow network at time $t$ accurately, it is necessary to compute all depressions that have been filled by time $t$ and "flood" them.

Liu and Snoeyink [18] (see also [5]) proposed an $O(n \log n)$-time algorithm under the SFD model that computes the fill times of all depressions assuming rain is falling at a constant rate on the entire terrain. In reality, localized extreme rainfall can affect downstream areas that do not receive heavy rainfall directly.

Lhomme et al. [17] developed a simplified flooding model, combining PDE-based models with ideas from the GIS community. They do so by utilizing a flow model similar to Liu and Snoeyink [18], as we do in our model. They divide the terrain into impact zones (a set of depressions) and determine in a preprocessing step the interaction between these zones. Then they model the 
flooding as a series of zones merging with adjacent flooded zones, and spilling excess water into adjacent zones. They incorporate additional parameters for the friction of the terrain. They compare their model to more traditional hydrological models and find that it had fairly reasonable accuracy (accurately predicting 56-98\% of the (non)-inundated areas) while improving performance significantly compared to baseline hydrological models (500-10,000 times faster).

Quinn et al. [22] proposed a framework for assigning multiple flow directions from each vertex in a terrain $\Sigma$, so that the proportion of water which flows from a vertex to a downslope neighbor is proportional to the gradient of the edge connecting the two. so it is important to model nonuniform rain events.

Arge et al. [2] described an I/O efficient algorithm to compute the related flow routing and flow accumulation problem under both the SFD and MFD models. The flow-routing problem asks what direction will water flow from any point on a terrain, and flow accumulation asks if water falls uniformly on the terrain how water flows over a each point in a terrain. They optimized their algorithms to be efficient for extremely large datasets.

Arge et al. [3] described an algorithm under the SFD model to compute the set of flooded vertices when a given volume of rain $\psi \geq 0$ falls on a given region $\mathcal{R}$. The running time of their algorithm is $O(n \log n)$. Their work raises a question whether $\Sigma$ can be preprocessed in a data structure so that a point-flood query can be answered quickly, say, in $O(\log n)$ time. This question was answered positively by Rav et al. [23]. They described data structures to compute flooding queries under the SFD model in $O(|\mathcal{R}| \log n)$ time. They also present an algorithm for answering flood queries that can handle uncertainty in data. Their algorithms, however, do not extend to the MFD model, as they very strongly use the tree structure of the water flow in the SFD model.

\section{CONCLUSION AND FUTURE WORK}

In this article, we have presented efficient data structures for answering flooding queries on a terrain $\Sigma$ under the multiflow-direction (MFD) model in which water at a point can flow along multiple downslope edges and which more accurately represent flooding events. In particular, we presented three main results: First, we presented an $O(n \log n)$-time algorithm to answer a terrainflood query: if it rains a volume $\psi$ according to a rain distribution $\mathcal{R}$, determine what regions of $\Sigma$ are flooded. Second, we presented an $O(n \log n+n m)$-time algorithm for preprocessing $\Sigma$ containing $m$ sinks into a data structure of size $O(\mathrm{~nm})$ for answering point-flood queries: given a rain distribution $\mathcal{R}$, a volume of rain $\psi$ falling according to $\mathcal{R}$, and point $q \in \Sigma$, one can determine in $O\left(|R| k+k^{2}\right)$ time whether $q$ is flooded, where $k$ is the number of maximal depressions in $\Sigma$ containing the query point $q$ and $|\mathcal{R}|$ is the number of vertices in $\mathcal{R}$ with positive rainfall. Finally, we presented algorithms for answering a flood-time query: given a rain distribution $\mathcal{R}$ and a point $q \in \Sigma$, determine the volume of rain that must fall before $q$ is flooded. Assuming that the product of two $k \times k$ matrices can be computed in $O\left(k^{\omega}\right)$ time, we showed that a flood-time query could be answered in $O\left(n k+k^{\omega}\right)$ time. We also presented an $\alpha$-approximation algorithm, for $\alpha>1$, that runs in $O\left(n \log n \log _{\alpha} \rho\right)$-time, where $\rho$ is a variable on $\Sigma$ that depends on the ratio between depression volumes.

Our work raises several open questions. There are a few obvious questions related to improving the performance of our algorithms. We mention two of them here:

(i) Can the ideas from Rav et al. [23] on flood-risk analysis under single-flow direction (SFD) model be used to develop a data structure for point-flood query with sublinear worst-case query time?

(ii) Can a flood-time query be answered in the same time (within a polylogarithmic factor) as a point-flood query? 
Besides these immediate questions, there are also a few high-level questions that we plan to investigate in the future: Our model of rain regions allows rain to be distributed arbitrarily among the vertices of the terrain without any restriction on the geographical structure of the rain region. In practice, the resolution of terrain models is typically much greater than the resolution of weather forecasts, meaning that a weather forecast will generally assign a similar or equal predicted rainfall amount to adjacent vertices of the terrain. Can the queries be answered more quickly when rain distribution is well behaved? Another interesting question is how quickly can we update the query results if the rain distribution changes dynamically? Finally, can our data structures be extended to incorporate uncertainty in the terrain height function or the rain distribution? As in [23], one could use a Monte Carlo approach to handle uncertainty in data, but it is not clear how to argue that only a small number of samples are sufficient to ensure high confidence in the output.

\section{ACKNOWLEDGMENTS}

We thank Alex Steiger for many thought provoking discussions, in particular sparking the idea of matrix multiplication for flood-time queries.

\section{REFERENCES}

[1] P. K. Agarwal, L. Arge, and K. Yi. 2006. I/O-efficient batched union-find and its applications to terrain analysis. In Proc. 22nd Annu. Sympos. on Comp. Geom. 167-176.

[2] L. Arge, J. S. Chase, P. Halpin, L. Toma, J. S. Vitter, D. Urban, and R. Wickremesinghe. 2003. Efficient flow computation on massive grid terrain datasets. GeoInformatica 7, 4 (2003), 283-313.

[3] L. Arge, M. Rav, S. Raza, and M. Revsbæk. 2017. I/O-efficient event based depression flood risk. In Proc. 19th Workshop on Algorithm Engineering and Experiments. 259-269.

[4] L. Arge and M. Revsbæk. 2009. I/O-efficient contour tree simplification. In Intl. Sympos. on Algos. and Computation. 1155-1165.

[5] L. Arge, M. Revsbæk, and N. Zeh. 2010. I/O-efficient computation of water flow across a terrain. In Proc. 26th Annu. Sympos. on Comp. Geom. 403-412.

[6] Norwegian Mapping Authority. 2013. Height DTM 10. https://kartkatalog.geonorge.no/metadata/kartverket/ dtm-10-terrengmodell-utm33/dddbb667-1303-4ac5-8640-7ec04c0e3918.

[7] Paul D. Bates and A. P. J. De Roo. 2000. A simple raster-based model for flood inundation simulation. Journal of Hydrology 236, 1-2 (2000), 54-77.

[8] H. Carr, J. Snoeyink, and U. Axen. 2003. Computing contour trees in all dimensions. Comp. Geom. 24, 2 (2003), 75-94.

[9] H. Carr, J. Snoeyink, and M. Panne. 2010. Flexible isosurfaces: Simplifying and displaying scalar topology using the contour tree. Comp. Geom. 43, 1 (2010), 42-58.

[10] Li-Chiu Chang, Hung-Yu Shen, and Fi-John Chang. 2014. Regional flood inundation nowcast using hybrid SOM and dynamic neural networks. Journal of Hydrology 519 (2014), 476-489.

[11] A. Danner, T. Mølhave, K. Yi, P. K. Agarwal, L. Arge, and H. Mitásová. 2007. TerraStream: From elevation data to watershed hierarchies. In Proc. 15th Annu. ACM Intl. Sympos. on Advances in GIS. 28.

[12] H. Edelsbrunner, J. Harer, and A. Zomorodian. 2001. Hierarchical Morse complexes for piecewise linear 2-manifolds. In Proc. 17th Annu. Sympos. Comp. Geom. 70-79.

[13] Bidur Ghimire, Albert S. Chen, Michele Guidolin, Edward C. Keedwell, Slobodan Djordjević, and Dragan A. Savić. 2013. Formulation of a fast 2D urban pluvial flood model using a cellular automata approach. Fournal of Hydroinformatics 15, 3 (2013), 676-686.

[14] Indiana Spatial Data Portal. 2013. Indiana Orthophotography (RGBI), LiDAR and Elevation. http://gis.iu.edu/ datasetInfo/statewide/in_2011.php.

[15] S. K. Jenson and J. O. Domingue. 1988. Extracting topographic structure from digital elevation data for geographic information system analysis. Photogrammetric Engineering and Remote Sensing 54, 11 (1988), 1593-1600.

[16] M. Van Kreveld, R. Oostrum, C. Bajaj, V. Pascucci, and D. Schikore. 1997. Contour trees and small seed sets for isosurface traversal. In Proc. 13th Annu. Sympos. on Comp. Geom. 212-220.

[17] Julien Lhomme, Paul Sayers, Ben Gouldby, Paul Samuels, Martin Wills, and Jonatan Mulet-Marti. 2008. Inundation modelling recent development and application of a rapid flood spreading method. In Flood Risk Management: Research and Practice. CRC Press, 30-39. 
[18] Y. Liu and J. Snoeyink. 2005. Flooding triangulated terrain. In Proc. 11th Intl. Sympos. on Spatial Data Handling. Springer, Berlin, 137-148.

[19] Aaron Lowe and Pankaj K. Agarwal. 2018. Flood-risk analysis on terrains under the multiflow-direction model. In Proce. 26th ACM SIGSPATIAL Int. Conf. Advan. Geograph. Inf. Syst. ACM, New York, 53-62.

[20] J. F. O'Callaghan and D. M. Mark. 1984. The extraction of drainage networks from digital elevation data. Computer Vision, Graphics, and Image Processing 28, 3 (1984), 323-344.

[21] Pennsylvania Spatial Data Access. 2008. PAMAP Program DEM Mosaics by Lidar Delivery Zones. http://www.pasda. psu.edu/uci/SearchResults.aspx?Keyword=PAMAP.

[22] P. F. B. J. Quinn, K. Beven, P. Chevallier, and O. Planchon. 1991. The prediction of hillslope flow paths for distributed hydrological modelling using digital terrain models. Hydrological Processes 5, 1 (1991), 59-79.

[23] M. Rav, A. Lowe, and P. K. Agarwal. 2017. Flood risk analysis on terrains. In Proc. of the 25th ACM SIGSPATIAL Int. Conference on Advances in GIS. ACM, New York, 36.

[24] S. P. Tarasov and M. N. Vyalyi. 1998. Construction of contour trees in 3D in O(n log n) steps. In Proc. 14th Annu. Sympos. on Comp. Geom. 68-75.

[25] Mahyat Shafapour Tehrany, Biswajeet Pradhan, Shattri Mansor, and Noordin Ahmad. 2015. Flood susceptibility assessment using GIS-based support vector machine model with different kernel types. Catena 125 (2015), 91-101.

[26] N. D. Volp, B. C. Van Prooijen, and G. S. Stelling. 2013. A finite volume approach for shallow water flow accounting for high-resolution bathymetry and roughness data. Water Resources Research 49, 7 (2013), 4126-4135.

Received December 2018; revised May 2019; accepted June 2019 